Preprint typeset in JHEP style. - HYPER VERSION

CECS-PHY-04/05

hep-th/0403179

\title{
Localized Intersections of Non-Extremal p-branes and S-branes
}

\author{
José D. Edelstein ${ }^{\dagger \natural}$, Javier Mas ${ }^{\ddagger}$ \\ ${ }^{\dagger}$ Instituto de Física de La Plata - Conicet, Universidad Nacional de La Plata \\ C.C. 67, (1900) La Plata, Argentina \\ ${ }^{\natural}$ Centro de Estudios Científicos (CECS), Casilla 1469, Valdivia, Chile \\ ${ }^{\ddagger}$ Departamento de Física de Partículas, Universidad de Santiago de Compostela \\ E-15782 Santiago de Compostela, Spain \\ edels@fisica.unlp.edu.ar, jamas@fpaxp1.usc.es
}

\begin{abstract}
A class of solutions to Supergravity in 10 or 11 dimensions is presented which extends the non-standard or semi-local intersections of $D p$-branes to the case of non-extremal p-branes. The type of non-extremal solutions involved in the intersection is free and we provide two examples involving black-branes and/or $D-\bar{D}$ systems. After a rotation among the time coordinate and a relatively transverse radial direction the solutions admit the interpretation of an intersection among D-branes and S-branes. We speculate on the relevance of these configurations both to study time dependent phenomena in the AdS/CFT correspondence as well as to construct cosmological brane-world scenarios within String Theory admitting accelerating expansion of the Universe.
\end{abstract}

Keywords: 5-branes, Brane Intersections, String Cosmology, Supergravity Solutions. 


\section{Contents}

1. Introduction 1

2. Ansatz and Separability of the Equations of Motion 4

3. Intersecting Black-Branes 9

4. Intersecting Black-branes and $D-\bar{D}$ system $\quad 12$

5. Intersecting $D$-branes and $S$-branes 15

6. Discussion and Prospects 17

A. Solving for Intersecting Black-Branes $\quad 18$

B. Solving for Black-branes intersecting $D-\bar{D}$ system 21

C. Bestiary of solutions 25

C.1 Solutions depending on two parameters 25

\begin{tabular}{ll}
\hline C.2 Solutions depending on one parameter & 26 \\
\hline
\end{tabular}

\section{Introduction}

That extremal $p$-brane supergravity solutions correspond to the macroscopic description of string theory $D p$-branes is a well established and fairly understood issue. This neat correspondence has been very fruitful in understanding aspects like the entropy of extremal black holes or the AdS/CFT correspondence. These objects admit a further description as solitons in the tachyonic field theory of unstable branes [1]. Regarding non-supersymmetric branes, whose stability is not even guaranteed, the precise identification among the open and closed string theory point of views is more subtle and disputable. Such is the case of black-branes, $D-\bar{D}$ systems or, more recently, a class of time dependent solutions generically termed $S$-branes [2] which have been the subject of intense analysis (see, for example, [3, 4, 5]). Being time dependent solutions, it is natural to conjecture that they may represent a macroscopic description of the rolling tachyon that drives the decay of unstable branes [2]. Still this interpretation, mainly due to the presence of singularities, is a subject of a

controversy [6, 7, 8] which is well beyond the scope of this paper. Therefore, just to better fix the nomenclature of this article, by $S$-branes we shall denote the aforementioned class of time dependent solutions to $D=10,11$ dimensional supergravity. We are confident that our construction is robust 
enough to encompass more general solutions, like the regular ones that are being announced while we type the last paragraphs of this paper [9].

In the present article we will study configurations admitting the interpretation of an intersection of $D$-branes and $S$-branes. Motivations to look for such a system can be gathered from different arenas. For example, from the rolling tachyon framework, the picture advocated in [2] can be generalized to asymmetric decays along a spacelike dimension $x$. Say, for different $\operatorname{sign}(x)= \pm 1$ the tachyon field, $T(x, t)$, could decay in opposite directions of the tachyon potential. The end process of such a behavior would be a timelike kink, identifiable with a $D p$-brane in the far future. Alternatively, one might think of a stable $D p$-brane intersecting an unstable $D\left(p^{\prime}+1\right)$-brane such that a tachyonic timelike kink in the worldvolume of the latter gives raise to an $S p^{\prime}$-brane that intersects the original stack of $D p$-branes. In a way or the other, it is clear that it is meaningful to consider a configuration that superimposes the tachyonic origin of $D$-branes and $S$-branes described above. The microscopic meaning of such a time dependent process deserves investigation, and whatever it is, one should also expect a macroscopic effective description coming from supergravity. In a first approximation, at least, the solutions we will present here have the correct symmetries and charges to be a good starting point. As well, known solutions corresponding to different kinds of branes can be recovered as particular limits of ours.

Leaving the rolling tachyon aside as an inspiring mechanism, one could just be interested in time dependent solutions to supergravity. In the literature these have mainly been worked out in connection with cosmology [10, 11, 12 More recently, some efforts have been devoted to understand and overcome the difficulties that prevent to embed an accelerating cosmology into a higher dimensional consistent framework like $M$ theory [13]. Mainly in [14] the importance of admitting time dependent compactifications was illustrated in a concrete example where the compactified manifold had constant negative curvature. It was a nice observation made in [15, 16] that this solution coincides precisely with a neutral supergravity $S M 2$-brane. An $S p$-brane has $p+1$ Euclidean dimensions. Hence the $S M 2$ yields precisely 3 dimensions which can account for the three dimensional space. The remnant outer space needs to be compactified. While this counting is fine for cosmology, from a particle physicist point of view there is little support to considering an $S M 2$-brane instead of any other $S p$-brane or, say, an $S M 5$-brane with 3 Euclidean longitudinal coordinates compactified on a torus, or even intersections thereof [17, 18]. While $D$-branes are known to trap gauge interactions along their worldvolume, it is less clear that $S$-branes work alike (the corresponding field theories should be Euclidean). If one is interested in a construction that performs both tasks, of trapping gauge interactions and having a time dependence, then an intersection between $D$-branes and $S$-branes looks promising.

The ideal situation would involve a stack of $D 3$-branes intersecting a space-filling neutral $S D 8$ brane. The metric would exhibit a double warping of the component functions. One in $r$, the radial distance to the position of the $D 3$-branes along the $S D 8$-brane Euclidean nine dimensional worldvolume. The other in $t$, the only coordinate outside the $S D 8$-brane which is placed exactly at a fixed time $t=t_{0}$. In a first approximation we would like to answer questions like: does a configuration like the previous one exist? Is the associated cosmology viable? Does it exhibit late time accelerated expansion? Are there other interesting examples? What are the general intersection rules and constraints? How many parameters are contained in the most general solution? Questions 
of a different nature also arise such as: are there time dependent processes in the quantum field theory living on the D3-branes that are dual to these solutions from the AdS/CFT point of view?

In order to make some progress along these lines it is convenient to take advantage from what is known about intersections [19], mainly of non-extremal $p$-branes [20, 21, 22]. Moreover, we are looking for solutions that depend on two coordinates $r$ and $t$. These will be the outer coordinates to the $D$ and $S$-branes respectively. This reminds of a similar setup affronted by the so called semi-localized (or non-standard) intersections [23, 24, 25]. However with the exception of reference [26] these intersections have always dealt with extremal branes. Moreover, the two coordinates were spacelike radial distances to the intersection region along the relative transverse directions. Therefore, on top of generalizing the ansatz to admit non-extremal solutions, we must include a signature freedom that allows us, at the end, to trade the time for one of these radial coordinates. In short, the ansatz we will analyze in the present paper contains the following line element

$$
d s^{2}=-\eta_{T} T^{2} d t^{2}+C^{2} d \vec{z}_{q}^{2}+\eta_{X} X^{2} d x^{2}+U^{2} d \Omega_{X, \sigma_{X}}^{2}+\eta_{Y} Y^{2} d y^{2}+V^{2} d \Omega_{Y, \sigma_{Y}}^{2}+W^{2} d \vec{w}_{p_{t}}^{2} .
$$

It has the correct symmetries to represent the intersection of a pair of branes. Call them $X$ and $Y$. We will refer to them generically with the index $A=X, Y$. Each p-brane has dimension $q_{A}=q+1+p_{A}$, thus we will also denote them as $q_{A}$-branes. $q$ is the dimension of the common Euclidean space where they intersect, which is spanned by cartesian coordinates $z_{1}, \ldots, z_{q}$. The coordinates $x, \theta_{X}^{1}, \ldots, \theta_{X}^{p_{X}}$ span the space along the $q_{X}$-brane and transverse relative to the $q_{Y}$-brane. Conversely, the $q_{Y}$-brane has coordinates $y, \theta_{Y}^{1}, \ldots, \theta_{Y}^{p_{Y}}$ along the space relatively transverse to the $X$-brane. The cartesian coordinates $w_{1}, \ldots, w_{p_{t}}$ span the overall transverse space. The angular variables $\theta_{A}^{1}, \ldots, \theta_{A}^{p_{A}}$ are coordinates of a maximally symmetric space with line element $d \Omega_{p_{A}, \sigma_{A}}^{2}$, i.e. $R_{\theta_{A}^{a} \theta_{A}^{b}}^{A}=\sigma_{A}\left(p_{A}-1\right) g_{\theta_{A}^{a} \theta_{A}^{b}}$, where $\sigma_{A}=+1,-1,0$ denotes respectively a spherical, hyperbolic or flat space. This ansatz must be supplemented with one for the forms respecting the isometries. All the functions will depend on both "radial" relatively transverse coordinates $x$ and $y$. A not minor assumption will be the factorizability of all the functions $\mathcal{F}(x, y)=\mathcal{F}_{x}(x) \mathcal{F}_{y}(y)$. (Subindices $x, y$ will generically refer in this paper to functions depending on those variables.) Eventually we will like to reinterpret one of them, say $x$, as a time. Therefore we have inserted phases $\eta_{T}, \eta_{X}, \eta_{Y}= \pm 1$. We will carry them all along the computations and thus postpone to the end the decision where to put the timelike signature. Of course, special attention should be paid to the appearance of imaginary charges and fluxes.

For $\eta_{T}=\eta_{X}=\eta_{Y}=1$, the extremal case analyzed in [24] is recovered in the special limit $T=C, X=U$ and $Y=V$ with $\sigma_{X}=\sigma_{Y}=+1$. Then $x$ and $y$ are the radial distances to the intersection region along the Euclidean $p_{X}+1$ and $p_{Y}+1$ dimensional relative transverse spaces. In [26] an intersection involving black-branes has been worked out. It will be reobtained in a later section as a particular case of our construction. By keeping track of the phases $\eta_{A}$, the great generality of our ansatz provides enough room to consider pairwise partially localized intersections of any of the following objects: $D$-branes, $E$-branes [27], black-branes, $S$-branes, $D-\bar{D}$ systems and Schwarzschild branes (neutral black-branes).

The paper is organized in the following form. In section 2 we will obtain the general form of the separated equations of motion. In obtaining this system the starting ansatz (1.1) must be supplemented by some further constraints. In the following three sections we shall exhibit particular 
solutions of the system, representing intersections of black-branes with black-branes (section 3), $D-\bar{D}$ systems (section 4 ) and $S$-branes (section 5 ; in this last case we actually focus in the extremal limit on the p-brane side). We obtain intersection rules for all these cases, the full system of algebraic equations that constrain the parameters of the corresponding solutions and we elaborate on some aspects of these configurations that might be relevant in the light of the motivations stated above. Section 6 includes the discussion and speculation on the relevance of these configurations to study time dependent phenomena in the AdS/CFT correspondence as well as to construct cosmological scenarios within String Theory admitting accelerating expansion of the Universe. Appendices A and $\mathrm{B}$ display, for completeness, details about the computations that lead to the final expressions given in the text. Appendix $\mathrm{C}$ exhibits a list of explicit solutions to the algebraic constraints given in section 3.

\section{Ansatz and Separability of the Equations of Motion}

We shall deal with the usual action for a generic dilatonic Einstein-Maxwell gravity theory in $D$ spacetime dimensions

$$
S=\frac{1}{16 \pi G_{D}} \int d^{D} x \sqrt{-g}\left(R-\frac{1}{2}(\partial \phi)^{2}-\sum_{A=1}^{Q} \frac{1}{2 n_{A} !} e^{a_{A} \phi} F_{n_{A}}^{2}\right),
$$

The action includes gravity, a dilaton and $Q$ field strengths of arbitrary form degree $n_{A} \leq D / 2$ and coupling to the dilaton $a_{A}$. We will eventually set $D=10,11$ and choose appropriate values of $a_{A}$ to make contact with $I I A, I I B$ and $11 d$ supergravity theories though there is clearly room to consider more general cases. The equations of motion can be written in the following form:

$$
\begin{aligned}
& R^{\mu}{ }_{\nu}=\frac{1}{2} \partial^{\mu} \phi \partial_{\nu} \phi+\sum_{A=1}^{Q} \frac{1}{2 n_{A} !} e^{a_{A} \phi}\left(n_{A} F^{\mu \rho_{2} \ldots \rho_{n}} F_{\nu \rho_{2} \ldots \rho_{n} A}-\frac{n_{A}-1}{D-2} F_{n_{A}}^{2} \delta^{\mu}{ }_{\nu}\right) . \\
& \frac{1}{\sqrt{-g}} \partial_{\mu}\left(\sqrt{-g} \partial^{\mu}\right) \phi=\sum_{A=1}^{Q} \frac{a_{A}}{2 n_{A} !} e^{a_{A} \phi} F_{n_{A}}^{2}, \\
& \partial_{\mu_{1}}\left(\sqrt{-g} e^{a_{A} \phi} F^{\mu_{1} \ldots \mu_{n_{A}}}\right)=0,
\end{aligned}
$$

supplemented with the Bianchi identities $\partial_{[\nu} F_{\left.\mu_{1} \ldots \mu_{n_{A}}\right]}=0$. Also, since we restrict to pairwise intersections, we shall set $Q=2$. For the metric functions, the ansatz shown in (1.1) will be used. Concerning the $R R$ forms, we have, in the electric frame, the following fluxes

$$
\begin{aligned}
F_{t, 1 \ldots q}^{X} \theta_{X}^{1} \ldots \theta_{X}^{p_{X}}{ }_{x y} & =\sqrt{\gamma\left(\theta_{X}^{a}\right)} \partial_{y} E^{X} \\
F_{t, 1 \ldots q}^{Y} \theta_{Y}^{1} \ldots \theta_{Y}^{p_{Y} y x} & =\sqrt{\gamma\left(\theta_{Y}^{a}\right)} \partial_{x} E^{Y}
\end{aligned}
$$

where $E^{A}(x, y)$ are the gauge potentials and $\gamma\left(\theta_{X}^{a}\right), \gamma\left(\theta_{Y}^{a}\right)$ are the volume factors of the corresponding maximally symmetric spaces. For the case of magnetic branes, these field strengths must be 
understood as the Hodge duals of the relevant ones,

$$
\tilde{F}_{A}^{\theta_{B}^{1} \ldots \theta_{B}^{p_{B}}}=\frac{1}{\left(D-n_{A}\right) !} \frac{e^{-a_{A} \phi}}{\sqrt{-g}} \epsilon^{\theta_{B}^{1} \ldots \theta_{B}^{p_{B}} \mu_{1} \ldots \mu_{n}} F_{\mu_{1} \ldots \mu_{n_{A}}}^{A} \quad A=X, Y
$$

As mentioned above, the factorization assumption states that $\mathcal{F}(x, y)=\mathcal{F}_{x}(x) \mathcal{F}_{y}(y)$ for all functions. Following [24] in order to bring the equations of motion into a manageable form, the following definitions will prove to be instrumental

$$
\begin{aligned}
f_{x}(x) & =x^{-p_{X}} T_{x} C_{x}^{q} U_{x}^{p_{X}} V_{x}^{p_{Y}} W_{x}^{p_{t}} X_{x}^{-1} Y_{x} \\
f_{y}(y) & =y^{-p_{Y}} T_{y} C_{y}^{q} U_{y}^{p_{X}} V_{y}^{p_{Y}} W_{y}^{p_{t}} X_{y} Y_{y}^{-1} \\
S^{X} & =\frac{W^{2 p_{t}} V^{2 p_{Y}}}{\sqrt{g}} e^{\epsilon_{X} a_{X} \phi}=\frac{W^{p_{t}}}{T C^{q} X Y} \frac{V^{p_{Y}}}{U^{p_{X}}} e^{\epsilon_{X} a_{X} \phi} \\
S^{Y} & =\frac{W^{2 p_{t}} U^{2 p_{X}}}{\sqrt{-g}} e^{\epsilon_{Y} a_{Y} \phi}=\frac{W^{p_{t}}}{T C^{q} X Y} \frac{U^{p_{X}}}{V^{p_{Y}}} e^{\epsilon_{Y} a_{Y} \phi}
\end{aligned}
$$

where $\epsilon_{A}$ is a positive (negative) sign that accounts for electric (magnetic) branes. With these ingredients, the diagonal Einstein equations read as follows

$$
\begin{gathered}
\square \ln T=\square \ln C=\eta\left(\delta_{\|}^{Y} \frac{S^{Y}}{\sqrt{g}}\left(\partial_{x} E^{Y}\right)^{2}+\delta_{\|}^{X} \frac{S^{X}}{\sqrt{g}}\left(\partial_{y} E^{X}\right)^{2}\right) \\
\square \ln X+\eta_{X} X^{-2}\left[\partial_{x}^{2}\left(\ln f x^{p_{X}}\right)-2 \partial_{x} \ln X \partial_{x}\left(\ln f x^{p_{X}}\right)+\left(\partial_{x} \ln T\right)^{2}+q\left(\partial_{x} \ln C\right)^{2}\right. \\
\left.+p_{X}\left(\partial_{x} \ln U\right)^{2}+p_{Y}\left(\partial_{x} \ln V\right)^{2}+p_{t}\left(\partial_{x} \ln W_{t}\right)^{2}+\left(\partial_{x} \ln Y\right)^{2}-\left(\partial_{x} \ln X\right)^{2}\right] \\
=\eta\left(\delta_{\|}^{Y} \frac{S^{Y}}{\sqrt{g}}\left(\partial_{x} E^{Y}\right)^{2}+\delta_{\|}^{X} \frac{S^{X}}{\sqrt{g}}\left(\partial_{y} E^{X}\right)^{2}\right)-\eta_{X} X^{-2}\left[\frac{1}{2}\left(\partial_{x} \phi\right)^{2}\right] \\
\square \ln Y+\eta_{Y} Y^{-2}\left[\partial_{y}^{2}\left(\ln f y^{p_{Y}}\right)-2 \partial_{y} \ln Y \partial_{y}\left(\ln f y^{p_{Y}}\right)+\left(\partial_{y} \ln T\right)^{2}+q\left(\partial_{y} \ln C\right)^{2}\right. \\
\left.+p_{X}\left(\partial_{y} \ln U\right)^{2}+p_{Y}\left(\partial_{y} \ln V\right)^{2}+p_{t}\left(\partial_{y} \ln W_{t}\right)^{2}+\left(\partial_{y} \ln X\right)^{2}-\left(\partial_{y} \ln Y\right)^{2}\right] \\
=\eta\left(\delta_{\|}^{Y} \frac{S^{Y}}{\sqrt{g}}\left(\partial_{x} E^{Y}\right)^{2}+\delta_{\|}^{X} \frac{S^{X}}{\sqrt{g}}\left(\partial_{y} E^{X}\right)^{2}\right)-\eta_{Y} Y^{-2}\left[\frac{1}{2}\left(\partial_{y} \phi\right)^{2}\right] \\
\square \ln U-\frac{\sigma_{X}\left(p_{X}-1\right)}{U^{2}}=\eta\left(\delta_{\perp}^{Y} \frac{S^{Y}}{\sqrt{g}}\left(\partial_{x} E^{Y}\right)^{2}+\delta_{\|}^{X} \frac{S^{X}}{\sqrt{g}}\left(\partial_{y} E^{X}\right)^{2}\right) \\
\square \ln V-\frac{\sigma_{Y}\left(p_{Y}-1\right)}{V^{2}}=\eta\left(\delta_{\|}^{Y} \frac{S^{Y}}{\sqrt{g}}\left(\partial_{x} E^{Y}\right)^{2}+\delta_{\perp}^{X} \frac{S^{X}}{\sqrt{g}}\left(\partial_{y} E^{X}\right)^{2}\right) \\
\square \ln W=\eta\left(\delta_{\perp}^{Y} \frac{S^{Y}}{\sqrt{g}}\left(\partial_{x} E^{Y}\right)^{2}+\delta_{\perp}^{X} \frac{S^{X}}{\sqrt{g}}\left(\partial_{y} E^{X}\right)^{2}\right)
\end{gathered}
$$

where $\eta \equiv \eta_{T} \eta_{X} \eta_{Y}$, and $\delta_{\|}^{A}, \delta_{\perp}^{A}$ are the typical exponents entering the harmonic superposition rule

$$
\delta_{\|}^{A}=\left(\frac{D-q_{A}-3}{2(D-2)}\right) \quad ; \quad \delta_{\perp}^{A}=\left(\frac{-q_{A}-1}{2(D-2)}\right) .
$$


The D'Alembertian is given by

$$
\square=\frac{1}{\sqrt{|G|}} \partial_{M}\left(\sqrt{|G|} \partial^{M}\right)=\frac{\eta_{X}}{X^{2}}\left[\partial_{x} \ln \left(f x^{p_{X}}\right) \partial_{x}+\partial_{x}^{2}\right]+\frac{\eta_{Y}}{Y^{2}}\left[\partial_{y} \ln \left(f y^{p_{Y}}\right) \partial_{y}+\partial_{y}^{2}\right] .
$$

In addition, there is a single off diagonal equation

$$
\begin{aligned}
R_{x y}= & -\partial_{x y}\left(\ln f x^{p_{X}} y^{p_{Y}}\right)+\partial_{x} \ln Y\left(\partial_{y} \ln f y^{p_{Y}}\right)+\partial_{y} \ln X\left(\partial_{x} \ln f x^{p_{X}}\right) \\
& -\partial_{x} \ln T \partial_{y} \ln T-q \partial_{x} \ln C \partial_{y} \ln C-p_{X} \partial_{x} \ln U \partial_{y} \ln U-p_{Y} \partial_{x} \ln V \partial_{y} \ln V \\
& -p_{t} \partial_{x} \ln W \partial_{y} \ln W+\partial_{x} \ln Y \partial_{y} \ln Y+\partial_{x} \ln X \partial_{y} \ln X-2 \partial_{x} \ln Y \partial_{y} \ln X \\
= & \frac{1}{2} \partial_{x} \phi \partial_{y} \phi
\end{aligned}
$$

where the first term straightforwardly vanishes from the factorization ansatz. As for the dilaton equation, we obtain

$$
\square \phi=-\frac{\eta}{2} \frac{\epsilon_{X} a_{X} S^{X}}{\sqrt{-g}}\left(\partial_{y} E^{X}\right)^{2}-\frac{\eta}{2} \frac{\epsilon_{Y} a_{Y} S^{Y}}{\sqrt{-g}}\left(\partial_{x} E^{Y}\right)^{2}
$$

Finally, the equations of motion satisfied by the gauge potentials are compactly written as

$$
\begin{aligned}
& \partial_{x}\left(S^{X}\left(\partial_{y} E^{X}\right)\right)=0 \quad ; \quad \partial_{y}\left(S^{Y}\left(\partial_{x} E^{Y}\right)\right)=0 \\
& \partial_{y}\left(S^{X}\left(\partial_{y} E^{X}\right)\right)=0 \quad ; \quad \partial_{x}\left(S^{Y}\left(\partial_{x} E^{Y}\right)\right)=0
\end{aligned}
$$

We shall start from here solving the full system. Invoking factorizability these equations can be integrated to give

$$
\begin{aligned}
& S_{x}^{X} E_{x}^{X}=l_{X} \quad ; \quad S_{y}^{Y} E_{y}^{Y}=l_{Y} \\
& S_{y}^{X}\left(\partial_{y} E_{y}^{X}\right)=c_{X} \quad ; \quad S_{x}^{Y}\left(\partial_{x} E_{x}^{Y}\right)=c_{Y}
\end{aligned}
$$

where $l_{A}$ and $c_{A}$ are constants. In passing by, and for later use, let us mention that the product $l_{A} c_{A}$ gives basically the RR-charge (density per unit $\mathrm{W}$ space volume)

$$
\begin{aligned}
& \mathcal{Q}_{X}=\int d \theta_{Y}^{1} \ldots d \theta_{Y}^{p_{Y}} \tilde{F}_{\theta_{Y}^{1} . . \theta_{Y}^{p_{Y}} w^{1} \ldots w_{p_{t}}}=\eta l_{X} c_{X} \Omega_{p_{Y}} \\
& \mathcal{Q}_{Y}=\int d \theta_{X}^{1} \ldots d \theta_{X}^{p_{X}} \tilde{F}_{\theta_{X}^{1} . . \theta_{X}^{p_{X}} w^{1} \ldots w_{p_{t}}}^{Y}=\eta l_{Y} c_{Y} \Omega_{p_{X}}
\end{aligned}
$$

with $\Omega_{p_{A}}=2 \pi^{\frac{p_{A}+1}{2}} / \Gamma\left(\frac{p_{A}+1}{2}\right)$. In order to proceed, let us insert the last two expressions in $(2.17)$ into the Einstein Equations. After multiplying by $X_{y}^{2} Y_{x}^{2}$ it is easy to recognize the conditions that must be imposed in order to achieve separability of the system. They are:

$$
\begin{aligned}
& \frac{X_{y}^{2}}{S_{y}^{Y} \sqrt{g}_{y}}=X_{y}^{2} W_{y}^{-2 p_{t}} U_{y}^{-2 p_{X}} e^{-\epsilon_{Y} a_{Y} \phi_{y}}=1 \\
& \frac{Y_{x}^{2}}{S_{x}^{X} \sqrt{g}_{x}}=Y_{x}^{2} W_{x}^{-2 p_{t}} V_{x}^{-2 p_{Y}} e^{-\epsilon_{X} a_{X} \phi_{x}}=1 .
\end{aligned}
$$


Notice that this set of conditions is the same as in 24 for the critical case and it is scalar under reparametrizations of either $x$ or $y$. Still, at this stage it is not clear whether they overdetermine the system or not, so they will have to be checked for consistency a posteriori. Now, it is not hard to realize that (2.20) and (2.21) are not sufficient in order to implement the separation of variables. From the angular $R^{\theta_{X}} \theta_{X}$ and $R^{\theta_{Y}} \theta_{Y}$ equations for $\ln U$ and $\ln V$, it is immediate to see that the following conditions are also needed:

$$
Y_{x}=V_{x} \quad ; \quad X_{y}=U_{y}
$$

This constraint (which is not necessary for $p_{X}$ or $p_{Y}=1$ ) is a natural one stating that the metric functions along each brane behave alike when separating along the relative transverse radial direction. After all these considerations, the separated equations adopt the following succinct form,

$$
\begin{aligned}
& \left(x^{p_{X}} f_{x}\left(\ln T_{x}\right)^{\prime}\right)^{\prime}=d_{\|}^{Y} E_{x}^{Y^{\prime}}+\kappa_{T} f_{x} x^{p_{X}} \\
& \left(x^{p_{X}} f_{x}\left(\ln C_{x}\right)^{\prime}\right)^{\prime}=d_{\|}^{Y} E_{x}^{Y^{\prime}}+\kappa_{C} f_{x} x^{p_{X}} \\
& \left(\ln X_{x}\right)^{\prime \prime}-\left(\ln f_{x} x^{p_{X}}\right)^{\prime}\left(\ln X_{x}\right)^{\prime}-\left(\ln X_{x}\right)^{\prime 2}+\left(\ln f_{x} x^{p_{X}}\right)^{\prime \prime}+\left(\ln T_{x}\right)^{\prime 2}+q\left(\ln C_{x}\right)^{\prime 2} \\
& \quad+p_{X}\left(\ln U_{x}\right)^{\prime 2}+\left(p_{Y}+1\right)\left(\ln V_{x}\right)^{\prime 2}+p_{t}\left(\ln W_{x}\right)^{\prime 2}=-\frac{1}{2}{\phi_{x}^{\prime}}^{2}+d_{\|}^{Y} \frac{E_{x}^{Y^{\prime}}}{f_{x} x^{p_{X}}}+\kappa_{X} \\
& \quad\left(x^{p_{X}} f_{x}\left(\ln Y_{x}\right)^{\prime}\right)^{\prime}=d_{\|}^{Y} E_{x}^{Y^{\prime}}+\kappa_{Y} f_{x} x^{p_{X}} \\
& \left(x^{p_{X}} f_{x}\left(\ln U_{x}\right)^{\prime}\right)^{\prime}=d_{\perp}^{Y} E_{x}^{Y^{\prime}}+\eta_{X} \sigma_{X}\left(p_{X}-1\right)\left(X_{x} / U_{x}\right)^{2} x^{p_{X}} f_{x}+\kappa_{U} f_{x} x^{p_{X}} \\
& \left(x^{p_{X}} f_{x}\left(\ln V_{x}\right)^{\prime}\right)^{\prime}=d_{\|}^{Y} E_{x}^{Y^{\prime}}+\kappa_{V} f_{x} x^{p_{X}} \\
& \left(x^{p_{X}} f_{x}\left(\ln W_{x}\right)^{\prime}\right)^{\prime}=d_{\perp}^{Y} E_{x}^{Y^{\prime}}+\kappa_{W} f_{x} x^{p_{X}} \\
& \left(x^{p_{X}} f_{x} \phi_{x}^{\prime}\right)^{\prime}=-e^{Y} E_{x}^{Y^{\prime}}+\kappa_{\phi} f_{x} x^{p_{X}}
\end{aligned}
$$

and, conversely,

$$
\begin{aligned}
& \left(y^{p_{Y}} f_{y}\left(\ln T_{y}\right)^{\prime}\right)^{\prime}=d_{\|}^{X} E_{y}^{X^{\prime}}-\kappa_{T} f_{y} y^{p_{Y}} \\
& \left(y^{p_{Y}} f_{y}\left(\ln C_{y}\right)^{\prime}\right)^{\prime}=d_{\|}^{X} E_{y}^{X^{\prime}}-\kappa_{C} f_{y} y^{p_{Y}} \\
& \left(\ln Y_{y}\right)^{\prime \prime}-\left(\ln f_{y} y^{p_{Y}}\right)^{\prime}\left(\ln Y_{y}\right)^{\prime}-\left(\ln Y_{y}\right)^{\prime 2}+\left(\ln f_{y} y^{p_{Y}}\right)^{\prime \prime}+\left(\ln T_{y}\right)^{\prime 2}+q\left(\ln C_{y}\right)^{\prime 2} \\
& \quad+p_{Y}\left(\ln V_{y}\right)^{\prime 2}+\left(p_{X}+1\right)\left(\ln U_{y}\right)^{\prime 2}+p_{t}\left(\ln W_{y}\right)^{\prime 2}=-\frac{1}{2} \phi_{y}^{\prime 2}+d_{\|}^{X} \frac{E_{y}^{X^{\prime}}}{f_{y} y^{p_{Y}}}-\kappa_{Y} \\
& \left(y^{p_{Y}} f_{y}\left(\ln X_{y}\right)^{\prime}\right)^{\prime}=d_{\|}^{X} E_{y}^{X^{\prime}}-\kappa_{X} f_{y} y^{p_{Y}} \\
& \left(y^{p_{Y}} f_{y}\left(\ln V_{y}\right)^{\prime}\right)^{\prime}=d_{\perp}^{X} E_{y}^{X^{\prime}}+\eta_{Y} \sigma_{Y}\left(p_{Y}-1\right)\left(Y_{y} / V_{y}\right)^{2} y^{p_{Y}} f_{y}-\kappa_{U} f_{y} y^{p_{Y}} \\
& \left(y^{p_{Y}} f_{y}\left(\ln U_{y}\right)^{\prime}\right)^{\prime}=d_{\|}^{X} E_{y}^{X^{\prime}}-\kappa_{V} f_{y} y^{p_{Y}} \\
& \left(y^{p_{Y}} f_{y}\left(\ln W_{y}\right)^{\prime}\right)^{\prime}=d_{\perp}^{X} E_{y}^{X^{\prime}}-\kappa_{W} f_{y} y^{p_{Y}} \\
& \left(y^{p_{Y}} f_{y} \phi_{y}^{\prime}\right)^{\prime}=-e^{X} E_{y}^{X^{\prime}}-\kappa_{\phi} f_{y} y^{p_{Y}}
\end{aligned}
$$


where we have defined, for convenience, the following constants

$$
d_{\|}^{A}=\tilde{c}_{A} \delta_{\|}^{A} \quad ; \quad d_{\perp}^{A}=\tilde{c}_{A} \delta_{\perp}^{A} \quad ; \quad e^{A}=\frac{1}{2} \tilde{c}_{A} \epsilon_{A} a_{A} \quad ; \quad \tilde{c}_{A}=\eta_{T} \eta_{A} l_{A}^{2} c_{A} \quad A=X, Y
$$

and the tilde denotes derivatives with respect to the only argument of each function. The original system has been successfully separated. There remains however a single equation that mixes both solutions: the off diagonal Einstein equation (2.14), or,

$$
\begin{aligned}
& \left(\ln Y_{x}\right)^{\prime}\left(\ln f_{y} y^{p_{Y}}\right)^{\prime}+\left(\ln X_{y}\right)^{\prime}\left(\ln f_{x} x^{p_{X}}\right)^{\prime}-\left(\ln T_{x}\right)^{\prime}\left(\ln T_{y}\right)^{\prime}-q\left(\ln C_{x}\right)^{\prime}\left(\ln C_{y}\right)^{\prime} \\
& -p_{X}\left(\ln U_{x}\right)^{\prime}\left(\ln U_{y}\right)^{\prime}-p_{Y}\left(\ln V_{x}\right)^{\prime}\left(\ln V_{y}\right)^{\prime}-p_{t}\left(\ln W_{x}\right)^{\prime}\left(\ln W_{y}\right)^{\prime} \\
& \quad+\left(\ln Y_{x}\right)^{\prime}\left(\ln Y_{y}\right)^{\prime}+\left(\ln X_{x}\right)^{\prime}\left(\ln X_{y}\right)^{\prime}-2\left(\ln Y_{x}\right)^{\prime}\left(\ln X_{y}\right)^{\prime}=\frac{1}{2} \phi_{x}^{\prime} \phi_{y}^{\prime}
\end{aligned}
$$

Since this equation does not contain second derivatives, it typically will impose constraints among the parameters and integration constants. The above system of equations contains contributions that are proportional to the separation constants $\kappa_{\alpha}$. These are genuine to the present setup, revealing the fact that we are dealing with an intersection. Although these constants may surely lead to interesting physics, they make the analytic integration far more involved. Therefore, at this stage, we shall set them all equal to zero, $\kappa_{\alpha}=0$, and defer a more general analysis for a later study. We will see that, even in this simplifying case, there is still a rich structure for the solutions of this system. With $\kappa_{\alpha}=0$, the separated systems (2.23) or (2.24) are nothing but a slight generalization of the one for a single $q_{A}$-brane, where the worldvolume global symmetry has been broken by the presence of the other brane (generically this just brings in additional integration constants subjected to some constraints).

The next step would be simply to specify the reader's favorite reduced ansatz, as well as a choice of $x$ and $y$ coordinates. Such choice needs not even be symmetric for both branes, as we shall show in a particular example. The presence of the other brane is still reflected in the off diagonal Einstein equation. Ideally one would like to borrow the most general p-brane solution from the literature, and see what constraints arise when coupling them through equation (2.26). In this paper we shall examine two cases. First we will select a generalized black-brane ansatz for both intervening branes. This case includes the solutions found in [26]. We provide general formulas for the integrated expressions and perform a full scan of the possible intersections. We will find that the presence of additional integration constants is essential to admit intersections where both horizons are nonzero. As a second example, we will affront an asymmetric ansatz where, while we keep for the $X$-brane the same black-brane-like solution, we use for the $Y$-brane the three parameter solution that has been obtained recently in connection with $S$-brane supergravity solutions [3, 17]. It is related by a coordinate transformation to the one obtained in Ref. 28] (see also [29]), where it was also found as part of a more general four parameter family (see also the appendix of [30] for another approach to a general solution). In an appealing analysis, this three parameter solution was identified with the supergravity description of a non BPS system of $N D p$-branes coinciding with $\bar{N} \bar{D} p$-branes [31]. The parameters involved characterize the mass, the net charge $($ for $N \neq \bar{N})$ and the vacuum expectation value of the tachyon. Therefore we will refer to this solution as the $D-\bar{D}$ system (see also the recent papers [32, 33] and references therein). 
Importantly enough, we will keep track of the phases $\eta_{A}$ in order to see what possibilities are at hand to select, say, $x$ as a time. From this, we will be able to present a family of solutions that represent intersections of $D$-branes and $S$-branes. Furthermore, the great generality of our ansatz allows to consider pairwise partially localized intersections of any of the following objects: $D$-branes, $E$-branes, black-branes, S-branes, $D-\bar{D}$ systems.

\section{Intersecting Black-Branes}

In this section we are going to present a family of solutions describing partially localized intersections of black-branes. We collect some of the details of the integration of this system in Appendix A. In considering intersections among black-branes, one is naturally led to the following ansatz

$$
\begin{aligned}
& T_{x}=C_{x} f_{x}^{1 / 2} \quad ; \quad X_{x}=\tilde{U}_{x} f_{x}^{-1 / 2} \quad ; \quad U_{x}=x \tilde{U}_{x} \quad ; \quad Y_{x}=V_{x} \\
& T_{y}=C_{y} f_{y}^{1 / 2} \quad ; \quad Y_{y}=\tilde{V}_{y} f_{y}^{-1 / 2} \quad ; \quad V_{y}=y \tilde{V}_{y} \quad ; \quad X_{y}=U_{y}
\end{aligned}
$$

which leads to the Schwarzschild-like line element (after omitting tildes)

$$
\begin{aligned}
d s^{2}= & C^{2}\left(-\eta_{T} f_{x} f_{y} d t^{2}+d \vec{z}_{q}^{2}\right)+U^{2}\left(\eta_{X} f_{x}^{-1} d x^{2}+x^{2} d \Omega_{p_{X}, \sigma_{X}}^{2}\right)+V^{2}\left(\eta_{Y} f_{y}^{-1} d y^{2}+y^{2} d \Omega_{p_{Y}, \sigma_{Y}}^{2}\right) \\
& +W^{2} d \vec{w}_{p_{t}}^{2}
\end{aligned}
$$

Inserting equations $(3.1)-(3.2)$ in $(2.7)-(2.8)$ result in the following gauge choices:

$$
\begin{aligned}
& 1=C_{x}^{q+1} U_{x}^{p_{X}-1} V_{x}^{p_{Y}+1} W_{x}^{p_{t}} \\
& 1=C_{y}^{q+1} U_{y}^{p_{X}+1} V_{y}^{p_{Y}-1} W_{y}^{p_{t}}
\end{aligned}
$$

It turns out that a solution exists if and only if $\eta_{A} \sigma_{A}=+1$, i.e. $d \Omega_{p_{A}, \sigma_{A}}^{2}$ being a spherical (hyperbolic) line element if and only if $x_{A}$ is a spacelike (timelike) coordinate (see Appendix A for details). The solution can be obtained in terms of two harmonic functions on each side

$$
\begin{aligned}
& f_{x}=1-\frac{2 \mu_{Y}}{x^{p_{X}-1}} \quad ; \quad f_{y}=1-\frac{2 \mu_{X}}{y^{p_{Y}-1}} \\
& H_{x}=1+\frac{Q_{Y}}{x^{p_{X}-1}} \quad ; \quad H_{y}=1+\frac{Q_{X}}{y^{p_{Y}-1}}
\end{aligned}
$$

The quantities $Q_{A}$ are, as usual, proportional to the number of $q_{A}$-branes whereas $\mu_{A}$ are the nonextremal black-brane parameters. The final expression for the metric functions and the dilaton are given by

$$
\begin{aligned}
& C_{x}=f_{x}^{\frac{c_{C}^{Y}}{2 \mu_{Y}\left(p_{X}-1\right)}} H_{x}^{-\frac{D-q_{Y}-3}{\Delta_{Y}}} \\
& C_{y}=f_{y}^{\frac{c_{C}^{X}}{2 \mu_{X}\left(p_{Y}-1\right)}} H_{y}^{-\frac{D-q_{X}-3}{\Delta_{X}}} \\
& U_{x}=f_{x}^{\frac{c_{U}^{Y}+2 \mu_{Y}}{2 \mu_{Y}\left(p_{X}-1\right)}} H_{x}^{\frac{q_{Y}+1}{\Delta_{Y}}} \\
& U_{y}=f_{y}^{\frac{c_{U}^{X}}{2 \mu_{X}\left(p_{Y}-1\right)}} H_{y}^{-\frac{D-q_{X}-3}{\Delta_{X}}} \\
& V_{x}=f_{x}^{\frac{c_{V}^{Y}}{2 \mu_{Y}\left(p_{X}-1\right)}} H_{x}^{-\frac{D-q_{Y}-3}{\Delta_{Y}}} \\
& V_{y}=f_{y}^{\frac{c_{V}^{X}+2 \mu_{X}}{2 \mu_{X}\left(p_{Y}-1\right)}} H_{y}^{\frac{q_{X}+1}{\Delta_{X}}} \\
& W_{x}=f_{x}^{\frac{c_{W}^{Y}}{2 \mu_{Y}\left(p_{X}-1\right)}} H_{x}^{\frac{q_{Y}+1}{\Delta_{Y}}} \\
& W_{y}=f_{y}^{\frac{c_{W}^{X}}{2 \mu_{X}\left(p_{Y}-1\right)}} H_{y}^{\frac{q_{X}+1}{\Delta_{X}}} \\
& e^{\phi_{x}}=f_{x}^{\frac{c_{\phi}^{Y}}{2 \mu_{Y}\left(p_{X}-1\right)}} H_{x}^{\frac{\epsilon_{Y} a_{Y}(D-2)}{\Delta_{Y}}} \\
& e^{\phi_{y}}=f_{y}^{\frac{c_{\phi}^{X}}{2 \mu_{X}\left(p_{Y}-1\right)}} H_{y}^{\frac{\epsilon_{X}^{a_{X}}(D-2)}{\Delta_{X}}}
\end{aligned}
$$


whereas for the gauge potentials $(2.5)-(2.6)$ we obtain

$$
\begin{aligned}
& E^{X}=E_{x}^{X} E_{y}^{X}=\eta_{T} \eta_{X} \sqrt{\eta_{T} \eta_{X} \frac{Q_{X}}{\left(Q_{X}+2 \mu_{X}\right)} \frac{2(D-2)}{\Delta_{X}}} x^{p_{X}} f_{x}^{\chi_{Y}} H_{x}^{\frac{2(D-2)}{\Delta_{Y}}} \frac{f_{y}}{H_{y}} \\
& E^{Y}=E_{x}^{X} E_{y}^{X}=\eta_{T} \eta_{Y} \sqrt{\eta_{T} \eta_{Y} \frac{Q_{Y}}{\left(Q_{Y}+2 \mu_{Y}\right)} \frac{2(D-2)}{\Delta_{Y}}} \frac{f_{x}}{H_{x}} y^{p_{Y}} f_{y}^{\chi_{X}} H_{y}^{\frac{2(D-2)}{\Delta_{X}}}
\end{aligned}
$$

where the values of the constants $\Delta_{A}$ and $\chi_{A},(A=X, Y)$ are given by

$$
\begin{aligned}
\Delta_{A} & =\left(q_{A}+1\right)\left(D-q_{A}-3\right)+\frac{1}{2}(D-2) a_{A}^{2} \\
\chi_{A} & =\frac{-1}{\mu_{A}\left(p_{B}-1\right)}\left(p_{t} c_{W}^{A}+p_{A} c_{V}^{A}-\left(c_{U}^{A}+2 \mu_{A}\right)+\frac{1}{2} \epsilon_{B} a_{B} c_{\phi}^{A}\right) \quad A \neq B
\end{aligned}
$$

For completeness, we have explicitly exhibited the phases $\eta_{A}$ in the expressions for the forms. We see that if we wanted to exchange the signatures of, say, $t$ and $x$ by setting $\eta_{T}=\eta_{X}=-1$ in an extremal solution the gauge potential $E^{Y}$ would become imaginary for $\eta_{Y}=+1$. This is the case of the so-called E-branes first discussed by Hull [27]. However, for a non zero value of the nonextremality parameter $\mu_{Y}$, still a real flux is possible by doing either $Q_{Y}$ or $\mu_{Y}$ negative in a certain interval, thus at the price of making the singularity naked. This seems to agree with the observation in [5] that just non-extremal $S$-branes (in this case the Y-brane) have a chance to admit real charges. The off diagonal Einstein equation constrains, as usual, the allowed intersections, through the same equation that was found for extremal solutions [24] ${ }^{1}$

$$
q+3=\frac{\left(q_{X}+1\right)\left(q_{Y}+1\right)}{(D-2)}-\frac{1}{2} \epsilon_{X} \epsilon_{Y} a_{X} a_{Y} .
$$

For completeness, we summarize here the general solution to this equation codified as $\left(q|X, Y| p_{t}\right)_{D}$,

$$
\begin{aligned}
& (1|M 5, M 5| 1)_{11} \\
& (1|N S 5, N S 5| 0)_{10} \\
& \left(q_{X}-3\left|D q_{X}, N S 5\right| 1\right)_{10} \quad 3 \leq q_{X} \leq 7 \\
& \left(\frac{q_{X}+q_{Y}}{2}-4\left|D q_{X}, D q_{Y}\right| 5-\frac{q_{X}+q_{Y}}{2}\right)_{10} \quad 8 \leq q_{X}+q_{Y} \leq 10
\end{aligned}
$$

where, in these expressions, we should remind that we are generally talking about non-extremal intersecting branes. In (3.8) there are twelve parameters $\mu_{A}, c_{C}^{A}, c_{U}^{A}, c_{V}^{A}, c_{W}^{A}, c_{\phi}^{A},(A=X, Y)$, subjected to nine constraints. Two of them arise from the gauge fixing condition (3.4)-(3.5)

$$
\begin{aligned}
& (q+1) c_{C}^{Y}+\left(p_{X}-1\right)\left(2 \mu_{Y}+c_{U}^{Y}\right)+\left(p_{Y}+1\right) c_{V}^{Y}+p_{t} c_{W}^{Y}=0 \\
& (q+1) c_{C}^{X}+\left(p_{Y}-1\right)\left(2 \mu_{X}+c_{V}^{X}\right)+\left(p_{X}+1\right) c_{U}^{X}+p_{t} c_{W}^{X}=0 .
\end{aligned}
$$

\footnotetext{
${ }^{1}$ We would like to remark that this intersection rule would not have emerged if we had considered, from the very beginning, that one of the intervening branes was neutral (Schwarzschild branes). In such a case, there is no intersection rule and solutions can be found roughly for any values of $q_{X}$ and $q_{Y}$.
} 
In the course of the integration the next four equations appear (see Appendix A)

$$
\begin{gathered}
\left(p_{X}-1\right) c_{U}^{Y^{2}}+2 \mu_{Y}\left(p_{X}-1\right)\left(c_{U}^{Y}+c_{C}^{Y}\right)+(q+1) c_{C}^{Y^{2}}+\left(p_{Y}+1\right) c_{V}^{Y^{2}}+p_{t} c_{W}^{Y}+\frac{1}{2} c_{\phi}^{Y^{2}}=0 \\
\left(p_{Y}-1\right) c_{V}^{X^{2}}+2 \mu_{X}\left(p_{Y}-1\right)\left(c_{V}^{X}+c_{C}^{X}\right)+(q+1) c_{C}^{X}+\left(p_{X}+1\right) c_{U}^{X}+p_{t} c_{W}^{X}+\frac{1}{2} c_{\phi}^{X^{2}}=0 \\
2\left(p_{X}-1\right)\left(2 \mu_{Y}+c_{U}^{Y}\right)+2 p_{t} c_{W}^{Y}+\epsilon_{Y} a_{Y} c_{\phi}^{Y}=0 \\
2\left(p_{Y}-1\right)\left(2 \mu_{X}+c_{V}^{X}\right)+2 p_{t} c_{W}^{X}+\epsilon_{X} a_{X} c_{\phi}^{X}=0
\end{gathered}
$$

From these, the first two are unavoidable. The second pair, however, is just a restriction on the phase space that allows to express the solution to the gauge potentials $E^{A}$ in terms of harmonic functions (3.6)-(3.7). Finally, from the off diagonal Einstein equation (2.26) we obtain the following three constraints ${ }^{2}$

$$
\begin{gathered}
p_{t} c_{W}^{Y}+\left(p_{Y}-1\right) c_{V}^{Y}+\frac{1}{2} \epsilon_{X} a_{X} c_{\phi}^{Y}=0 \\
p_{t} c_{W}^{X}+\left(p_{X}-1\right) c_{U}^{X}+\frac{1}{2} \epsilon_{Y} a_{Y} c_{\phi}^{X}=0 \\
\mu_{X} \mu_{Y}\left(p_{X}-1\right)\left(p_{Y}-1\right)+\mu_{X}\left(p_{Y}-1\right)\left(c_{V}^{Y}+c_{C}^{Y}\right)+\mu_{Y}\left(p_{X}-1\right)\left(c_{U}^{X}+c_{C}^{X}\right) \\
+(q+1) c_{C}^{X} c_{C}^{Y}+\left(p_{X}-1\right) c_{U}^{X} c_{U}^{Y}+\left(p_{Y}-1\right) c_{V}^{Y} c_{V}^{X}+p_{t} c_{W}^{Y} c_{W}^{X}+2 c_{U}^{X} c_{V}^{Y}+\frac{1}{2} c_{\phi}^{Y} c_{\phi}^{X}=0
\end{gathered}
$$

Let us pause here to comment about the generic features that solutions to this algebraic system exhibit. First of all, notice that there is always a simple solution of the first eight constraints given by $c_{\alpha}^{A}=0$ for all $\alpha$ except $c_{U}^{Y}=-2 \mu_{Y}$ and $c_{V}^{X}=-2 \mu_{X}$. With this, the last equation (3.26) reads just $\left(p_{X}-1\right)\left(p_{Y}-1\right) \mu_{X} \mu_{Y}=0$. Hence one of the two branes has to be set to extremality, either $\mu_{X}=0$ or $\mu_{Y}=0$. Within this class of solutions, the non-extremalization follows the simple recipe that was found for the case of standard intersections in [20]. In summary, generically we always find the two solutions in $D=10$

$$
\begin{aligned}
\left(\mu_{X}=0\right) \quad d s^{2}= & H_{x}^{\frac{q_{X}+1}{8}} H_{y}^{\frac{q_{Y}+1}{8}}\left[H_{x}^{-1} H_{y}^{-1}\left(-f_{x} d t^{2}+d \vec{z}_{q}^{2}\right)+H_{y}^{-1}\left(f_{x}^{-1} d x^{2}+x^{2} d \Omega_{p_{X}, 1}^{2}\right)\right. \\
& \left.+H_{x}^{-1}\left(d y^{2}+y^{2} d \Omega_{p_{Y}, 1}^{2}\right)+d \vec{w}_{p_{t}}^{2}\right] \\
\left(\mu_{Y}=0\right) \quad d s^{2}= & H_{x}^{\frac{q_{Y}+1}{8}} H_{y}^{\frac{q_{X}+1}{8}}\left[H_{x}^{-1} H_{y}^{-1}\left(-f_{y} d t^{2}+d \vec{z}_{q}^{2}\right)+H_{y}^{-1}\left(d x^{2}+x^{2} d \Omega_{p_{X}, 1}^{2}\right)\right. \\
& \left.+H_{x}^{-1}\left(f_{y}^{-1} d y^{2}+y^{2} d \Omega_{p_{Y}, 1}^{2}\right)+d \vec{w}_{p_{t}}^{2}\right]
\end{aligned}
$$

and the corresponding ones in $D=11$ for (3.14) with $\left(q_{A}+1\right) / 8$ replaced by $2 / 3$. We will refer to these as the "minimal non-extremal" deformations. However the system (3.18)-(3.26) admits also sometimes non-minimal extensions. In all the cases listed in (3.16), except for $q_{X}=3$, the solution depends on three parameters, two of which can be taken as $\mu_{X}, \mu_{Y}$. In all the remaining cases in $D=10$, notice that either $q=0$ or $p_{t}=0$ and there is a reduction in the number of parameters (since correspondingly $c_{C}^{Y}, c_{C}^{X}$ or $c_{W}^{Y}, c_{W}^{X}$ are absent). Also the case $(1|M 5, M 5| 1)$ has no dilaton and there are no $c_{\phi}^{Y}, c_{\phi}^{X}$. These situations never occur simultaneously (except for the cases $(0|D 3, D 5| 1)$

\footnotetext{
${ }^{2}$ For the case in which $X(Y)$ is a neutral brane, the constraint (3.25) (alternatively (3.24)) would be absent.
} 
and $(0|D 3, N S 5| 1)$ where, nevertheless, there is still a one parameter solution at the end), so in all these cases there are nine equations for ten variables, and we would expect a one parameter family of solutions. Solving the first eight constraints (3.18)-(3.25) for all the constants except $\mu_{X}$ and $\mu_{Y}$, yields typically four solutions which one can introduce into the quadratic equation (3.26), which then collapses to an identity of the sort $A \mu_{X} \mu_{Y}=0$ where $A$ is a numerical constant specific to each case. If $A \neq 0$ this equation implies, as before, either $\mu_{X}=0$ or $\mu_{Y}=0$. However, frequently, two out of the four solutions have $A=0$ and, therefore, automatically solve the last constraint. In these cases both $\mu_{X}$ and $\mu_{Y}$ are free and we obtain a 2-parameter family of solutions.

Summarizing, the constraints are always solved if one of the constituents is BPS. Most cases also admit an intersection among two non-extremal branes. Also notice that for each D3 or M5brane involved in the intersection, we must skip the corresponding $c_{\phi}^{A}$ as well. We have summarized, in Appendix $C$, the complete expressions for all possible intersections. Two of them agree with the ones found in [26]. One can see that, whereas the $H_{x}$ and $H_{y}$ follow a harmonic superposition rule, there is no such a pattern to encode the way the $f$ functions appear, aside from the interesting fact that always one of the branes is either $B P S$ or its "minimal non-extremal" deformation. It is possible, at this stage, to choose different values for the phases $\eta_{A}$ to obtain intersections involving $S$ branes. However, the ansatz we are dealing with does break $S O(1, q)$ isometries in the intersection. This would lead to non-isotropic $S$-branes. We are not going to study those configurations in this paper. Let us only mention that the kind of solutions involving non-isometric $S$-branes includes a family of completely regular solutions that might deserve further study [4].

\section{Intersecting Black-branes and $D-\bar{D}$ system}

As mentioned above, by $D-\bar{D}$ system we refer to a non-extremal solution that does not break the $S O(1, q)$ symmetry of the worldvolume. A general solution of this kind can be found in [28, 31]. This solution can be nicely fitted within our approach. However, we prefer to borrow the solution given in [3, 15] which is physically equivalent since both are related by a change of coordinates. The reason for this preference is twofold. On one side it is much simpler, and on the other it will allow us, in the upcoming section, to relate this configuration to another one which can be interpreted as an intersection of $D$-branes with $S$-branes.

To fix up the notation we will chose $X$ to be the black-brane and $Y$ the $D-\bar{D}$ system. For $X$ we adopt the same ansatz as in the previous section, therefore its integration follows closely the steps given in Appendix A after replacing $x \rightarrow y, X \rightarrow Y$ and $U \rightarrow V$. For the $Y$-brane, instead, we impose $T_{x}=C_{x}$ which implements the desired $S O(1, q)$ symmetry (as far as the $Y$-brane is concerned). The line element will acquire the following mixed structure

$$
\begin{aligned}
d s^{2}=C_{x}^{2} C_{y}^{2} & \left(-\eta_{T} f_{y} d t^{2}+d \vec{z}_{q}^{2}\right)+U_{y}^{2}\left(\eta_{X} X_{x}^{2} d x^{2}+U_{x}^{2} d \Omega_{p_{X}, \sigma_{X}}^{2}\right)+V_{x}^{2} V_{y}^{2}\left(\eta_{Y} f_{y}^{-1} d y^{2}+y^{2} d \Omega_{p_{Y}, \sigma_{Y}}^{2}\right) \\
& +W^{2} d \vec{w}_{p_{t}}^{2}
\end{aligned}
$$

The $S O(1, q)$ symmetry is broken by the black-brane $X$ through the non-extremal function $f_{y}$. Following [3, 15] the $x$ coordinate gauge fixing that leads to the solutions we are interested in, is given by $f_{x} x^{p_{X}}=1$, or, looking at (2.7), by $1=C_{x}^{1+q} U_{x}^{p_{X}} V_{x}^{p_{Y}+1} W_{x}^{p_{t}} X_{x}^{-1}$. With this, one notices that 
the left hand side of the Einstein equations (2.23) simplifies considerably. Following these papers it is fairly straightforward to carry out the integration which the reader can find for completeness in Appendix B (with the careful inclusion of the phases $\eta_{A}$ ). The solution reads:

$$
\begin{aligned}
C_{x} & =e^{c_{C}^{Y} x} K_{x}^{-\frac{D-q_{Y}-3}{\Delta_{Y}}} & C_{y} & =f_{y}^{\frac{c_{C}^{X}}{2 \mu_{X}\left(p_{Y}-1\right)}} H_{y}^{-\frac{D-q_{X}-3}{\Delta_{X}}} \\
X_{x} & =e^{c_{X}^{Y} x+p_{X} g(x)} K_{x}^{\frac{q_{Y}+1}{\Delta Y}} & X_{y} & =U_{y} \\
U_{x} & =e^{c_{U}^{Y} x+g(x)} K_{x}^{\frac{q_{Y}+1}{\Delta_{Y}}} & U_{y} & =f_{y}^{\frac{c_{U}^{X}}{\left.\mu_{X} p_{Y}-1\right)}} H_{y}^{-\frac{D-q_{X}-3}{\Delta_{X}}} \\
V_{x} & =e^{c_{V}^{Y} x} K_{x}^{-\frac{D-q_{Y}-3}{\Delta_{Y}}} & V_{y} & =f_{y}^{\frac{c_{V}^{X}+2 \mu_{X}}{2 \mu_{X}\left(p_{Y}-1\right)}} H_{y}^{\frac{q_{X}+1}{\Delta}} \\
W_{x} & =e^{c_{W}^{Y} x} K_{x}^{\frac{q_{Y}+1}{\Delta_{Y}}} & W_{y} & =f_{y}^{\frac{c_{W}^{X}}{2 \mu_{X}\left(p_{Y}-1\right)}} H_{y}^{\frac{q_{X}+1}{\Delta_{X}}} \\
e^{\phi_{x}} & =e^{c_{\phi}^{Y} x} K_{x}^{\frac{(D-2) a_{Y} \epsilon_{Y}}{\Delta_{Y}}} & e^{\phi_{y}} & =f_{y}^{\frac{c_{\phi}^{X}}{2 \mu_{X}\left(p_{Y}-1\right)}} H_{y}^{\frac{\epsilon_{X} a_{X}(D-2)}{\Delta_{X}}}
\end{aligned}
$$

with $f_{y}, H_{y}$ as given in (3.6) and (3.7), $\eta_{Y} \sigma_{Y}=+1$, and

$$
K_{x}=\frac{1}{2}\left(e^{2 \gamma_{Y}\left(x-x_{Y}\right)}-\zeta_{Y}\right) \quad e^{g(x)}=\left\{\begin{array}{cc}
\left(\frac{\beta}{\cosh \left(\left(p_{X}-1\right) \beta\left(x-x_{0}\right)\right)}\right)^{\frac{1}{p_{X}-1}} & \eta_{X} \sigma_{X}=-1 \\
e^{ \pm \beta\left(x-x_{0}\right)} & \sigma_{X}=0 \\
\left(\frac{\beta}{\sinh \left(\left(p_{X}-1\right) \beta\left(x-x_{0}\right)\right)}\right)^{\frac{1}{p_{X}-1}} & \eta_{X} \sigma_{X}=1
\end{array}\right.
$$

In these expressions, we have introduced two new parameters $\zeta_{Y}$ and $\gamma_{Y}$,

$$
\begin{aligned}
\zeta_{Y} & =\operatorname{sign}\left(\tilde{c}_{Y} \gamma_{Y}\right)=\eta_{T} \eta_{Y} \operatorname{sign}\left(c_{Y} \gamma_{Y}\right) \\
\gamma_{Y} & =(1+q) c_{C}^{Y}+\left(1+p_{Y}\right) c_{V}^{Y}-\frac{1}{2} \epsilon_{Y} a_{Y} c_{\phi}^{Y} .
\end{aligned}
$$

Notice that $\gamma_{Y}$ will turn out to be related to the $R R$ charge (the analog of $Q_{Y}$ in the previous ansatz). We will make the choice of $c_{Y}$ such that $\operatorname{sign}\left(c_{Y} \gamma_{Y}\right)=+1$ and therefore everywhere we may substitute $\eta_{T} \eta_{Y}$ in place of $\zeta_{Y}$. In the above solution, the independent parameters for the $x$ dependence can be taken to be $c_{C}^{Y}, c_{V}^{Y}, c_{W}^{Y}, c_{\phi}^{Y}$ and $x_{0}$. In the context of ordinary non BPS branes with $c_{C}^{Y}=c_{V}^{Y}$ (hence full $I S O\left(1+q+p_{Y}\right)$ ) and $c_{W}^{Y}=0$, this would be a three parameter family. Also the asymptotic value of the dilaton has been selected to absorb an additional constant $c_{\phi}^{Y^{\prime}}$, and $x_{Y}$ can be fixed at will by an appropriate choice of the origin for $x$. We have also made particular scale transformation on the coordinates to absorb additional constants (see Appendix B). The rest 
of the parameters appearing in (4.2) and (4.3) are derived from the previous ones:

$$
\begin{aligned}
c_{U}^{Y}= & c_{X}^{Y}=-\frac{1}{p_{X}-1}\left[(1+q) c_{C}^{Y}+\left(1+p_{Y}\right) c_{V}^{Y}+p_{t} c_{W}^{Y}\right] \\
\left(\beta^{Y}\right)^{2}= & \frac{1}{p_{X}\left(p_{X}-1\right)}\left[\frac{1}{p_{X}-1}\left((1+q) c_{C}^{Y}+\left(p_{Y}+1\right) c_{V}^{Y}+p_{t} c_{W}^{Y}\right)^{2}\right. \\
& \left.+(1+q)\left(c_{C}^{Y}\right)^{2}+\left(p_{Y}+1\right)\left(c_{V}^{Y}\right)^{2}+p_{t}\left(c_{W}^{Y}\right)^{2}+\frac{1}{2}\left(c_{\phi}^{Y}\right)^{2}\right]
\end{aligned}
$$

Actually, these two equations replace (3.18) and (3.20) in the previous ansatz, where now $\beta_{Y}$ plays the rôle of the non-extremality parameter.

From the off diagonal Einstein equation we obtain the same intersection rule as before (3.13) and algebraic constraints (3.24) and (3.25), which are sufficient to ensure the validity of the separability ansatz (2.20) and (2.21). Instead of (3.26), in the present setting we get

$$
\begin{gathered}
\left(p_{Y}-1\right) \mu_{X}\left(c_{C}^{Y}+c_{V}^{Y}\right)+(1+q) c_{C}^{X} c_{C}^{Y}+\left(p_{Y}-1\right) c_{V}^{X} c_{V}^{Y}+\left(p_{X}-1\right) c_{U}^{X} c_{U}^{Y} \\
+p_{t} c_{W}^{X} c_{W}^{Y}+2 c_{U}^{X} c_{V}^{Y}+\frac{1}{2} c_{\phi}^{X} c_{\phi}^{Y}=0
\end{gathered}
$$

In the present case, however, we do not have the analog of (3.22). This was simply a restriction imposed in order to be able to obtain harmonic solutions in the black-brane ansatz. This is not needed here. On the other side, the $X$ brane constants $c_{\alpha}^{X}$ are still bound by constraints (3.19), $(\sqrt[3.21]{)})$ and $(3.23)$. In total there is one algebraic equation less and, therefore, we do not need to set one of the branes to extremality, nor we expect to find discrete sets of solutions like the ones listed in Appendix C.

The final expressions for the gauge potentials are

$$
\begin{aligned}
& E^{X}=E_{x}^{X} E_{y}^{X}=e^{\kappa_{Y} x} K_{x}^{\frac{2(D-2)}{\Delta_{Y}}} \sqrt{\eta_{T} \eta_{X} \frac{Q_{X}}{\left(Q_{X}+2 \mu_{X}\right)} \frac{2(D-2)}{\Delta_{X}}} \frac{f_{y}}{H_{y}} \\
& E^{Y}=E_{x}^{Y} E_{y}^{Y}=-\eta_{T} \eta_{Y} \sqrt{\frac{2(D-2)}{\Delta_{Y}}} \frac{e^{2 \gamma_{Y}\left(x-x_{Y}\right)}}{K_{x}} y^{p_{Y}} f_{y}^{\chi_{X}} H_{y}^{\frac{2(D-2)}{\Delta_{X}}}
\end{aligned}
$$

with $\kappa_{Y}=-\frac{2}{p_{X}-1}\left(p_{X} p_{t} c_{W}^{Y}+\left(p_{X} p_{Y}+1\right) c_{V}^{Y}+(1+q) c_{C}^{Y}\right)+\epsilon_{X} a_{X} c_{\phi}^{Y}$. The corresponding charge densities (per unit $W$ space volume) are given by

$$
\begin{aligned}
& \mathcal{Q}_{X}=\eta_{T} \eta_{X} \eta_{Y}\left(p_{Y}-1\right) \sqrt{\eta_{T} \eta_{X} Q_{X}\left(2 \mu_{X}+Q_{X}\right) \frac{2(D-2)}{\Delta_{X}}} \Omega_{p_{Y}} \\
& \mathcal{Q}_{Y}=\eta_{T} \eta_{X} \eta_{Y} \sqrt{\frac{2(D-2)}{\Delta_{Y}}} \gamma_{Y} \Omega_{p_{X}}
\end{aligned}
$$

whence we see that $\gamma_{Y}$ is directly related to the charge. Contrary to the results in the previous section, notice that there is no obstruction now against a signature flip of the sort $\eta_{T}=\eta_{X}=-1$, which will be the subject of study in what follows. 


\section{Intersecting $D$-branes and $S$-branes}

In this section, the results obtained in the previous one will be exploited to generate intersection among supergravity $D$-branes and $S$-branes through analytic continuation ${ }^{3}$. To fix the conventions, we will take $X$ for the $D$-brane, and $Y$ as the $S$-brane. The solution can be obtained from the previous section exchanging the roles of $t$ and $x$ as timelike coordinates, by setting $\eta_{T}=\eta_{X}=-1$ and keeping $\eta_{Y}=+1$. Recall that the first ansatz applied to the $D$-brane $X$, (i.e. to the $y$ dependence) asks for $\sigma_{Y}=+1$. Moreover the usefulness of the second ansatz, as applied to the $x$ dependence, becomes apparent. Indeed, starting from (4.1) and setting the black-brane $X$ to criticality ${ }^{4} f_{y} \rightarrow 1$, we may rename $t \rightarrow z_{q+1}$ as well as $x \rightarrow t$ and $y \rightarrow r$ and thus end up with a line element of the form

$$
d s^{2}=C^{2} d \vec{z}_{p}^{2}-X^{2} d t^{2}+U^{2} d \Omega_{p_{D}, \sigma_{D}}^{2}+V^{2}\left(d r^{2}+r^{2} d \Omega_{p_{S},+1}^{2}\right)+W^{2} d \vec{w}_{p_{t}}^{2}
$$

where, in order to stress that $X$ is the $D$-brane and $Y$ the $S$-brane we have changed labels $X \rightarrow D$ and $Y \rightarrow S$. Now $p=q+1$ is the dimension of the Euclidean intersection manifold. Just to better grasp where the $S$-branes and the $D$-branes are one may momentarily set $X_{r}=C_{r}$ and $V_{t}=C_{t}$, which is a consistent truncation that only amounts to tuning the integration constants to $c_{U}^{D}=c_{C}^{D}$, as well as $c_{V}^{S}=c_{C}^{S}$. To recover the $D$-brane just skip all dependencies along its worldvolume coordinate $t$ (and remember that $U_{r}=X_{r}$ and $V_{t}=Y_{t}$ from (2.22))

$$
d s_{D}^{2}=C_{r}^{2}\left(-d t^{2}+d \vec{z}_{p}^{2}+d \Omega_{p_{D}, \sigma_{D}}^{2}\right)+V_{r}^{2}\left(d r^{2}+r^{2} d \Omega_{p_{S},+1}^{2}\right)+W_{r}^{2} d \vec{w}_{p_{t}}^{2}
$$

For $\sigma_{D}=0$ this is just the standard solution for a $D q_{D}$-brane with $q_{D}=\left(1+q+p_{D}\right)$, which is smeared along a $p_{t}$ dimensional outer space. The remnant of the presence of the intersecting $S$ brane is imprinted in the breaking of the $S O\left(1, p+p_{D}\right)$ symmetry, which allows us to regard other possibilities for the curvature $\sigma_{D}$ other than +1 . Conversely, to recover an $S\left(q_{S}=p+p_{S}\right)$-brane, we shall tune all functions $\mathcal{F}_{r} \rightarrow 1$, thus obtaining the following line element:

$$
d s_{S}^{2}=-X_{t}^{2} d t^{2}+C_{t}^{2}\left(d \vec{z}_{p}^{2}+d r^{2}+r^{2} d \Omega_{p_{S},+1}^{2}\right)+U_{t}^{2} d \Omega_{p_{D}, \sigma_{D}}^{2}+W_{t}^{2} d \vec{w}_{p_{t}}^{2}
$$

One may go, of course, to Euclidean coordinates $d z_{1}, \ldots, d z_{1+q_{S}}$ if desired. In accordance with the standard convention for a $S q_{S}$-brane [2, 3], (5.3) contains a $q_{S}+1$ dimensional isotropic "Euclidean worldvolume". For completeness, we reproduce here the full solution for the ansatz (5.1), leaving

\footnotetext{
${ }^{3}$ Notice that the name $D$-brane is being used for any extremal object. This is clearly an abuse of terminology, since we are also considering configurations involving $N S$ and $M$-theory branes

${ }^{4}$ We might also treat within our general framework, intersections involving $D-\bar{D}$ systems or black-branes with $S$-branes. The actual solutions can be easily obtained, following the lines of this section, by borrowing results from sections 3 and 4 of this paper.
} 
the details of its obtention to Appendix B,

$$
\begin{aligned}
X & =e^{c_{U} t} K_{t}^{\frac{q_{S}+1}{\Delta_{S}}} e^{p_{D} g(t)} H_{r}^{-\frac{d-q_{D}-3}{\Delta_{D}}} \\
C & =e^{c_{C} t} K_{t}^{-\frac{d-q_{S}-3}{\Delta_{S}}} H_{r}^{-\frac{d-q_{D}-3}{\Delta_{D}}} \\
U & =e^{c_{U} t} K_{t}^{\frac{q_{S}+1}{\Delta_{S}}} e^{g(t)} H_{r}^{-\frac{d-q_{D}-3}{\Delta_{D}}} \\
V & =e^{c_{V} t} K_{t}^{-\frac{d-q_{S}-3}{\Delta_{S}}} H_{r}^{\frac{q_{D}+1}{\Delta_{D}}} \\
W & =e^{c_{W} t} K_{t}^{\frac{q_{S}+1}{\Delta_{S}}} H_{r}^{\frac{q_{D}+1}{\Delta_{D}}} \\
e^{\phi} & =e^{c_{\phi} t} K_{t}^{\frac{\epsilon_{S} a_{S}(d-2)}{\Delta_{S}}} H_{r}^{\frac{\epsilon_{D} a_{D}(d-2)}{\Delta_{A}}} \\
K_{t} & =\frac{1}{2}\left(e^{2 \gamma_{S}\left(t-t_{S}\right)}+1\right) \quad ; \quad H_{r}=1+\frac{Q_{D}}{r^{p_{D}-1}}
\end{aligned}
$$

where we use $d$ for the dimensionality of spacetime to avoid confusion with the $D$-brane label. On the other hand, the fluxes are given by

$$
\begin{aligned}
& E^{D}=e^{\kappa_{D} t} K_{t}^{\frac{2(d-2)}{\Delta_{S}}} \sqrt{\frac{2(d-2)}{\Delta_{D}}}\left(\frac{1}{H_{r}}\right) \\
& E^{Y}=\sqrt{\frac{2(d-2)}{\Delta_{S}}} \frac{e^{2 \gamma_{S}\left(t-t_{S}\right)}}{K_{t}} r^{p_{S}} H_{r}^{\frac{2(d-2)}{\Delta_{D}}}
\end{aligned}
$$

The integration constants satisfy the same constraints as in the previous section. Also the intersection rule can be inferred from (3.13). It is preferable to re-express it directly in terms of the data that define the intersection, namely, the dimensions $q_{D}$ and $q_{S}$ of the intervening branes and $p$ of the intersection manifold

$$
p+2=\frac{\left(q_{D}+1\right)\left(q_{S}+1\right)}{d-2}-\frac{1}{2} \epsilon_{D} \epsilon_{S} a_{D} a_{S}
$$

The set of solutions is summarized in the following table

$$
\begin{array}{r}
(2|M 5, S M 5| 1)_{11} \\
(2|N S 5, S N S 5| 0)_{10} \\
\left(q_{D}-2\left|D q_{D}, S N S 5\right| 1\right)_{10} \\
\left(q_{S}-2\left|N S 5, S D q_{S}\right| 1\right)_{10} \\
\left(\frac{q_{D}+q_{S}}{2}-3\left|D q_{D}, S D q_{S}\right| 5-\frac{q_{D}+q_{S}}{2}\right)_{10} \quad 6 \leq q_{D}+q_{S} \leq 10
\end{array}
$$


in the notation $\left(p\left|D q_{D}, S q_{S}\right| p_{t}\right)_{d}$. One should not forget the limitation in the total number of dimensions, given by $d=1+p+p_{D}+\left(1+p_{S}\right)+p_{t}=2+q_{D}+q_{S}-p+p_{t}$.

One important reason to think of (5.7)-(5.8) as a new intersection rule is that it encompasses the interesting case $p=0$ which cannot be obtained by an analytic continuation from a semi-local timelike intersection (where it would force us to start with $q=-1$ ). This case has to be solved separately from scratch, and the final answer is given by the above solution after setting $p=0$. The set of solutions presented in (5.4)-(5.8) provides a supergravity description of intersecting $D$-branes and $S$-branes.

\section{Discussion and Prospects}

In this paper we have explored a family of supergravity solutions with the correct isometries to represent pairwise partially localized intersections of any pair of the following objects: $D$-branes, $E$-branes, black-branes, $S$-branes, $D-\bar{D}$ systems and Schwarzschild branes. We have developed in full detail the cases corresponding to intersection of black-branes with black-branes or a $D-\bar{D}$ system, and that of $S$-branes intersecting $D$-branes. These solutions are of the factorized type, a key assumption for achieving the separation of the equations of motion. We have put to zero the separation constants, and this is certainly a particular case that makes the integration feasible. Certainly an analysis of solutions where this is not so is a straightforward extension of the present work.

After rotating one radial coordinate and the time, each of these intersection yields p-brane solution with a time dependence of the $S$-brane type. Applications to cosmology should proceed as follows. First of all, identify among the factor spaces, a three dimensional maximally symmetric one lying inside the $D$-brane, and compactify all the rest. After going over to the Einstein frame, a specific four dimensional cosmological evolution emerges automatically. In this respect, let us mention that time dependent compactifications involving direct product of several factor spaces may lead to inflation with a sufficient number of e-foldings 34 (contrary to what happens whenever there is a single outer space [35], where a transient period of accelerated expansion is achieved but the number of e-foldings is around 1; see also [36, 37] for related results and generalizations). In our framework, however, the way these factor spaces appear is tightly constrained by the intersection rules and the need of an $\operatorname{ISO}(3)$ symmetric subspace. A detailed analysis of the cosmological features of these solutions will appear soon 38

Another avenue for future research has to do with the possibility to formulate AdS/CFT duality in a time dependent context. In order to do so, the near horizon geometries have to be carefully unravelled and, for that, a regular supergravity $S$-brane solution is probably needed. The method presented in this paper attempts to be a first step in that direction. It would be interesting to examine the very recent regular $S$-brane solutions found in 9] within this context. Of course, issues like the near horizon limit of such time dependent configurations are highly non-trivial. It would also be interesting to see whether the addition of tachyonic matter changes the singularity patterns of the configurations discussed in this paper or not. In this respect, we would like to point out that the most natural configuration to study these issues displays $D 3$-branes intersecting space-filling 
neutral $S D 8$-branes, and it is precisely for the latter that singularity theorems seem to be elusive [6, 7].

The microscopic description of the intersections of $D$-branes and $S$-branes might shed light into many of these issues and is worth studying.

Note added: Soon after submission of this manuscript, configurations involving S-branes and (space filling) D-branes have also been considered in studying a possible mechanism to resolve the aforementioned S-brane supergravity solutions' singularities [39].

\section{Acknowledgments}

We would like to express our gratitude to Max Bañados, Roberto Emparan, Martin Kruczenski, Alfonso Ramallo, Jorge Russo, Kostas Skenderis and Stefan Theisen for helpful comments and/or correspondence. JDE and JM are pleased to thank respectively the Theory Group in Santiago de Compostela and the Physics Laboratory at CECS for most warm hospitality while this work was being done. We both wish to thank the Benasque Center for Science where it was initiated.

This work has been supported in part by MCyT, FEDER and Xunta de Galicia under grant BFM2002-03881, by Fundación Antorchas, by the FCT (Portugal) grant POCTI/FNU/38004/2001, and by the EC Commission under the FP5 grant HPRN-CT-2002-00325. Institutional support to the Centro de Estudios Científicos (CECS) from Empresas CMPC is gratefully acknowledged. CECS is a Millennium Science Institute and is funded in part by grants from Fundación Andes and the Tinker Foundation.

\section{A. Solving for Intersecting Black-Branes}

In this appendix we shall give the details of the general solution used in the text. The procedure follows the constructive approach of [22] but we try to keep as general as possible. We shall integrate the dependence on one of the coordinates $x$. It should be noted that, regarding the $x$ radial coordinate living inside the $X$ brane, all the dependence on it comes from the presence of the $Y$ brane. Therefore the integration constants carry an index $Y$. The analog equations for $y$ dependence can be obtained after replacing $x \rightarrow y, X \rightarrow Y$ and $U \rightarrow V$. After inserting the gauge fixing ansatz (3.1) into (2.23) all the equations except the ones for $X_{x}$ may be integrated once to give

$$
\begin{aligned}
f_{x}\left(\ln T_{x}\right)^{\prime} & =\frac{d_{\|}^{Y} E_{x}^{Y}+c_{T}^{Y}}{x^{p_{X}}} & f_{x}\left(\ln V_{x}\right)^{\prime} & =\frac{d_{\|}^{Y} E_{x}^{Y}+c_{V}^{Y}}{x^{p_{X}}} \\
f_{x}\left(\ln C_{x}\right)^{\prime} & =\frac{d_{\|}^{Y} E_{x}^{Y}+c_{C}^{Y}}{x^{p_{X}}} & f_{x}\left(\ln W_{x}\right)^{\prime} & =\frac{d_{\perp}^{Y} E_{x}^{Y}+c_{W}^{Y}}{x^{p_{X}}} \\
f_{x}\left(\ln x U_{x}\right)^{\prime} & =\frac{d_{\perp}^{Y} E_{x}^{Y}+c_{U}^{Y}}{x^{p_{X}}}+\frac{\eta_{X} \sigma_{X}}{x} & f_{x} \phi_{x}^{\prime} & =\frac{-e^{Y} E_{x}^{Y}+c_{\phi}^{Y}}{x^{p_{X}}}
\end{aligned}
$$


Substituting $T_{x}=C_{x} f_{x}^{1 / 2}$ and subtracting from the equation for $C_{x}$, the following solution for $f_{x}$ is readily found

$$
f_{x}=1-\frac{2 \mu_{Y}}{x^{p_{X}-1}}
$$

with $c_{T}^{Y}=\mu_{Y}\left(p_{X}-1\right)+c_{C}^{Y}$. Notice, however, that it is also possible to find a differential equation for $f_{x}$ by adding up the following Einstein equations: $(q+1) R_{i}^{i}+\left(p_{X}-1\right) R^{\theta_{x}} \theta_{x}+\left(p_{Y}+1\right) R^{\theta_{y}} \theta_{y}+p_{t} R^{w}{ }_{w}$,

$$
\left(p_{X}-1\right)\left(f_{x} x^{p_{X}-1}\right)^{\prime}-\eta_{X} \sigma_{X}\left(p_{X}-1\right)^{2} x^{p_{X}-2}=0
$$

since the right hand side adds up to $\left(\left(q+1+p_{Y}+1\right) d_{\|}^{Y}+\left(p_{X}-1-p_{t}\right) d_{\perp}^{Y}\right) E^{\prime}=0$. The solution of this equation, with the right asymptotics, $f_{x}(\infty) \rightarrow 1$, is (A.2), with the additional constraint

$$
\sigma_{X} \eta_{X}=1
$$

The same reasoning, of course, applies to the Y brane. The gauge fixing constraints (3.4) (3.5) impose the following equality

$$
(q+1) c_{C}^{Y}+\left(p_{X}-1\right)\left(2 \mu_{Y}+c_{U}^{Y}\right)+\left(p_{Y}+1\right) c_{V}^{Y}+p_{t} c_{W}^{Y}=0
$$

with an analog expression for the other brane. In order to achieve the full integration we cast the equation (2.23) for $X_{x}$ in the following form

$$
\begin{aligned}
& f_{x}\left(f_{x}\left(\ln x U_{x}\right)^{\prime}\right)^{\prime}-\left(f_{x}^{\prime}+\frac{p_{X}-2}{x} f_{x}\right)\left(f_{x}\left(\ln x U_{x}\right)^{\prime}\right)+\frac{1}{2} f_{x} f_{x}^{\prime \prime}+\frac{1}{2}\left(\frac{p_{X}}{x} f_{x}-\frac{1}{2} f_{x}^{\prime}\right) f_{x}^{\prime} \\
& +\left(f_{x} \ln T_{x}\right)^{\prime 2}+q\left(f_{x} \ln C_{x}^{\prime}\right)^{2}+\left(p_{X}-1\right)\left(f_{x}\left(\ln x U_{x}\right)^{\prime}\right)^{2}+\left(p_{Y}+1\right)\left(f_{x} \ln V_{x}^{\prime}\right)^{2}+p_{t}\left(f_{x} \ln W_{x}^{\prime}\right)^{2} \\
& \quad=-\frac{1}{2}\left(f_{x} \phi_{x}^{\prime}\right)^{2}+d_{\|}^{Y} \frac{f_{x} E_{x}^{Y^{\prime}}}{x^{p_{X}}}
\end{aligned}
$$

Inserting the first integrals, the term which is independent of $E_{x}^{Y}$ yields the following algebraic constraint

$$
\left(p_{X}-1\right) c_{U}^{Y 2}+2 \mu_{Y}\left(p_{X}-1\right)\left(c_{U}^{Y}+c_{C}^{Y}\right)+(q+1) c_{C}^{Y 2}+\left(p_{Y}+1\right) c_{V}^{Y 2}+p_{t} c_{W}^{Y 2}+\frac{1}{2} c_{\phi}^{Y 2}=0
$$

The $E_{x}^{Y}$ dependent terms, on the other hand, yield the following equation

$$
\left[\left(\frac{f_{x}}{E_{x}^{Y}}\right)^{\prime}-\frac{\Gamma_{Y}}{x^{p_{X}} E_{x}^{Y}}+\frac{1}{x^{p_{X}}}\left(\frac{\tilde{c}_{Y}}{2}\right) \frac{\Delta_{Y}}{(D-2)}\right] E_{x}^{Y^{2}}=0
$$

with

$$
\begin{aligned}
& \Gamma^{Y}=2\left(p_{X}-1\right)\left(2 \mu_{Y}+c_{U}^{Y}\right)+2 p_{t} c_{W}^{Y}+\epsilon_{Y} a_{Y} c_{\phi}^{Y} \\
& \Delta_{Y}=\left(q_{Y}+1\right)\left(D-q_{Y}-3\right)+\frac{1}{2}(D-2) a_{Y}^{2}
\end{aligned}
$$


Analytic integration may be easily achieved in the particular case that we set $\Gamma_{Y}=0$. This is really a reduction by one in the number of parameters the solution may depend on. It would be interesting to lift this constraint in order to solve for the most general case. Now, for $\Gamma_{Y}=0$, the resulting equation for $E_{x}^{Y}$ can be solved as

$$
E_{x}^{Y}=\left(\frac{Q_{Y}\left(p_{X}-1\right)}{\tilde{c}_{Y}} \frac{2(D-2)}{\Delta_{Y}}\right) \frac{f_{x}}{H_{x}}
$$

in terms of a harmonic function $H_{x}$ in relative transverse space along the $X$ brane

$$
H_{x}=1+\frac{Q_{Y}}{x^{p_{X}-1}}
$$

With these ingredients we can readily obtain the integrated expressions

$$
\begin{aligned}
C_{x} & =f_{x}^{\frac{c_{C}^{Y}}{2 \mu_{Y}\left(p_{X}-1\right)}} H_{x}^{-\frac{D-q_{Y}-3}{\Delta_{Y}}} \\
X_{x} & =U_{x} f_{x}^{-1 / 2} \\
U_{x} & =f_{x}^{\frac{c_{U}^{Y}+2 \mu_{Y}}{2 \mu_{Y}\left(p_{X}-1\right)}} H_{x}^{\frac{q_{Y}+1}{\Delta_{Y}}} \\
Y_{x}=V_{x} & =f_{x}^{\frac{c_{V}^{Y}}{2 \mu_{Y}\left(p_{X}-1\right)}} H_{x}^{-\frac{D-q_{Y}-3}{\Delta_{Y}}} \\
W_{x} & =f_{x}^{\frac{c_{W}^{Y}}{2 \mu_{Y}^{\left(p_{X}-1\right)}}} H_{x}^{\frac{q_{Y}+1}{\Delta_{Y}}} \\
e^{\phi_{x}} & =f_{x}^{\frac{c_{\phi}^{Y}}{2 \mu_{Y}\left(p_{X}-1\right)}} H_{x}^{\frac{\epsilon_{Y} a_{Y}(D-2)}{\Delta_{Y}}}
\end{aligned}
$$

which are enough to compute the functions $S_{x}^{A}$. Using (2.17) we obtain

$$
E_{x}^{X}=\frac{l_{X}}{S_{x}^{X}}=l_{X} x^{p_{X}} f_{x}^{\chi_{Y}} H_{x}^{\frac{2(D-2)}{\Delta_{Y}}}
$$

with

$$
\chi_{Y}=\frac{-1}{\mu_{Y}\left(p_{X}-1\right)}\left(p_{t} c_{W}^{Y}+p_{Y} c_{V}^{Y}-\left(c_{U}^{Y}+2 \mu_{Y}\right)+\frac{1}{2} \epsilon_{X} a_{X} c_{\phi}^{Y}\right)
$$

Also, we may compute $S_{x}^{Y}\left(\partial_{x} E_{x}^{Y}\right)$ and equate it to $c_{Y}$ as in (2.17). Using (2.25) is enough to solve for the product $c_{Y} l_{Y}$ as

$$
c_{Y} l_{Y}=\left(p_{X}-1\right) \sqrt{\eta_{T} \eta_{Y} Q_{Y}\left(2 \mu_{Y}+Q_{Y}\right) \frac{2(D-2)}{\Delta_{Y}}}
$$


This can be inserted back into (A.10) to find

$$
E_{x}^{Y}=\frac{\eta_{T} \eta_{Y}}{l_{Y}} \sqrt{\eta_{T} \eta_{Y} \frac{Q_{Y}}{\left(2 \mu_{Y}+Q_{Y}\right)} \frac{2(D-2)}{\Delta_{Y}}} \frac{f_{x}}{H_{x}}
$$

Concerning the off diagonal Einstein equation, writing down $f x^{p_{X}} y^{p_{Y}} R_{x y}$ explicitly, we may separate the coefficients of different powers of $E_{x}^{Y}$ and $E_{y}^{X}$. The terms proportional to $E^{X} E^{Y}$ yield the same intersection rule as in the extremal case

$$
q+3=\frac{\left(q_{X}+1\right)\left(q_{Y}+1\right)}{(D-2)}-\frac{1}{2} \epsilon_{X} \epsilon_{Y} a_{X} a_{Y}
$$

From the terms proportional to $E^{Y}$ and $E^{X}$ we obtain the following constraints

$$
\begin{aligned}
& p_{t} c_{W}^{Y}+\left(p_{Y}-1\right) c_{V}^{Y}+\frac{1}{2} \epsilon_{X} a_{X} c_{\phi}^{Y}=0 \\
& p_{t} c_{W}^{X}+\left(p_{X}-1\right) c_{U}^{X}+\frac{1}{2} \epsilon_{Y} a_{Y} c_{\phi}^{X}=0
\end{aligned}
$$

and finally from the independent terms

$$
\begin{gathered}
\mu_{X} \mu_{Y}\left(p_{X}-1\right)\left(p_{Y}-1\right)+\mu_{X}\left(p_{Y}-1\right)\left(c_{V}^{Y}+c_{C}^{Y}\right)+\mu_{Y}\left(p_{X}-1\right)\left(c_{U}^{X}+c_{C}^{X}\right)+(q+1) c_{C}^{X} c_{C}^{Y} \\
+\left(p_{X}-1\right) c_{U}^{X} c_{U}^{Y}+\left(p_{Y}-1\right) c_{V}^{X} c_{V}^{Y}+p_{t} c_{W}^{X} c_{W}^{Y}+2 c_{U}^{X} c_{V}^{Y}+\frac{1}{2} c_{\phi}^{X} c_{\phi}^{Y}=0
\end{gathered}
$$

One can easily check that these equations are enough to verify the separability assumption (2.20) and (2.21).

The case with $p_{X}=1$ can also be worked along the same lines. In this case, the $Y$ brane is a co-dimension 2 defect inside the $X$ brane and therefore the harmonic functions involve logarithms of $x$. It is always understood that a completely analogous analysis goes through for the $y$ dependence.

\section{B. Solving for Black-branes intersecting $D-\bar{D}$ system}

For the second $D-\bar{D}$ system we will solve again only for the $x$ dependence, caused as before by the $Y$ brane. Also here, we shall follow a generalization of the approach in [17]. On top of $T=C$, the gauge fixing constraint will be taken to be $f_{x} x^{p_{X}}=1$, or equivalently

$$
1=C_{x}^{p} U_{x}^{p_{X}} V_{x}^{p_{Y}+1} W_{x}^{p_{t}} X_{x}^{-1}
$$


Now $p=q+1$ and $q_{A}=p+p_{A}$ is the dimension of each brane. The $x$ system can be first-integrated, except for the equations involving $X$ and $U$

$$
\begin{aligned}
& \left(\ln C_{x}\right)^{\prime}=d_{\|}^{Y} E_{x}^{Y}+c_{C}^{Y} \\
& \begin{aligned}
\left(\ln X_{x}\right)^{\prime \prime}-\left(\ln X_{x}\right)^{\prime 2}+p\left(\ln C_{x}\right)^{\prime 2}+p_{X}\left(\ln U_{x}\right)^{\prime 2}+\left(p_{Y}+1\right)\left(\ln V_{x}\right)^{\prime 2}+p_{t}\left(\ln W_{x}\right)^{\prime 2} \\
\quad=-\frac{1}{2}{\phi_{x}^{\prime}}^{2}+d_{\|}^{Y} E_{x}^{Y^{\prime}}
\end{aligned} \\
& \left(\ln U_{x}\right)^{\prime \prime}-\eta_{X} \sigma_{X}\left(p_{X}-1\right)\left(\frac{X_{x}}{U_{x}}\right)^{2}=d_{\perp}^{Y} E_{x}^{Y^{\prime}} \\
& \left(\ln V_{x}\right)^{\prime}=d_{\|}^{Y} E_{x}^{Y}+c_{V}^{Y} \\
& \left(\ln W_{x}\right)^{\prime}=d_{\perp}^{Y} E_{x}^{Y}+c_{W}^{Y} \\
& \phi_{x}^{\prime}=-e^{Y} E_{x}^{Y}+c_{\phi}^{Y}
\end{aligned}
$$

Let us define, for conciseness,

$$
\begin{aligned}
\sum_{\alpha} \ln U_{x \alpha} & =p \ln C_{x}+\left(p_{Y}+1\right) \ln V_{x}+p_{t} \ln W_{x} \\
\sum_{\alpha} c_{\alpha}^{Y} & =p c_{C}^{Y}+\left(p_{Y}+1\right) c_{V}^{Y}+p_{t} c_{W}^{Y} \\
\sum_{\alpha \| Y} c_{\alpha}^{Y} & =p c_{C}^{Y}+\left(p_{Y}+1\right) c_{V}^{Y}
\end{aligned}
$$

The non-extremality resides now in the distinction among $\ln X$ and $\ln U$. Following [2, 18, 17], we will parameterize this through a new function

$$
g=\frac{\ln X_{x}-\ln U_{x}}{p_{X}-1}
$$

Recalling the constraint (B.1) it follows that

$$
\begin{aligned}
& \ln U_{x}=g-\frac{1}{p_{X}-1} \sum_{\alpha} \ln U_{x \alpha} \\
& \ln X_{x}=p_{X} g-\frac{1}{p_{X}-1} \sum_{\alpha} \ln U_{x \alpha}
\end{aligned}
$$

and taking derivatives

$$
\begin{aligned}
& \left(\ln U_{x}\right)^{\prime}=g^{\prime}+d_{\perp}^{Y} E_{x}^{Y}+c_{U}^{Y} \\
& \left(\ln X_{x}\right)^{\prime}=p_{X} g^{\prime}+d_{\perp}^{Y} E_{x}^{Y}+c_{X}^{Y}
\end{aligned}
$$


where $c_{X}=c_{U}=-\frac{1}{p_{X}-1} \sum_{\alpha} c_{\alpha}$. After inserting these expressions into equation (B.3), a differential equation for $g$ is found

$$
g^{\prime \prime}-\eta_{X} \sigma_{X}\left(p_{X}-1\right) e^{2\left(p_{X}-1\right) g}=0
$$

with the solution

$$
g(x)=\left\{\begin{array}{cl}
\frac{1}{p_{X}-1} \ln \frac{\beta}{\cosh \left(\left(p_{X}-1\right) \beta\left(x-x_{0}\right)\right)} & \eta_{X} \sigma_{X}=-1 \\
\frac{1}{p_{X}-1} \ln \frac{\beta\left(x-x_{0}\right)}{\sinh \left(\left(p_{X}-1\right) \beta\left(x-x_{0}\right)\right)} & \sigma_{X}=0 \\
\sigma_{X}=1
\end{array}\right.
$$

From this result it is clear that $\beta$ plays the rôle of the non-extremality parameter. Indeed the limit $\beta \rightarrow 0$ is well defined only for the cases $\sigma_{X}=0$ and $\eta_{X} \sigma_{X}=1$, leading to a flat relatively transverse $p_{X}+1$ dimensional space. obtain

Noticing that $g^{\prime \prime}-\left(p_{X}-1\right)\left(g^{\prime}\right)^{2}=-\left(p_{X}-1\right) \beta^{2}$ we may insert this into equation (B.2) and

$$
\begin{gathered}
-p_{X}\left(p_{X}-1\right) \beta^{2}+\left(d_{\perp}^{Y}-d_{\|}^{Y}\right) E_{x}^{Y^{\prime}}+\left(p_{X}-1\right)\left(d_{\perp}^{Y} E_{x}^{Y}-\sum_{\alpha} \frac{c_{\alpha}^{Y}}{p_{X}-1}\right)^{2}+p\left(d_{\|}^{Y} E_{x}^{Y}+c_{C}^{Y}\right)^{2} \\
+\left(p_{Y}+1\right)\left(d_{\|}^{Y} E_{x}^{Y}+c_{V}^{Y}\right)^{2}+p_{t}\left(d_{\perp}^{Y} E_{x}^{Y}+c_{W}^{Y}\right)^{2}+\frac{1}{2}\left(-e^{Y} E_{x}^{Y}+c_{\phi}^{Y}\right)^{2}=0
\end{gathered}
$$

From the constant term we find

$$
\frac{1}{p_{X}-1}\left(\sum_{\alpha} c_{\alpha}^{Y}\right)^{2}+\sum_{\alpha} c_{\alpha}^{Y^{2}}+\frac{1}{2} c_{\phi}^{Y^{2}}=p_{X}\left(p_{X}-1\right) \beta^{2}
$$

and from the $E_{x}^{Y}$ dependent part,

$$
\left(\frac{1}{E_{x}^{Y}}\right)^{\prime}+\frac{2 \gamma_{Y}}{E_{x}^{Y}}=-\frac{\Delta_{Y} \tilde{c}_{Y}}{2(D-2)}
$$

where $\Delta_{Y}$ is the same as in $($ A.9 $)$ and

$$
\gamma_{Y}=\sum_{\alpha \| Y} c_{\alpha}^{Y}-\frac{1}{2} a_{Y} c_{\phi}^{Y}
$$

Equation $(\mathbb{B} .13)$ can be readily integrated as follows

$$
E_{x}^{Y}=-\frac{2(D-2)}{\Delta_{Y}} \frac{\gamma_{Y}}{\tilde{c}_{Y}} \frac{e^{2 \gamma_{Y}\left(x-x_{Y}\right)}}{K_{x}}
$$

with

$$
K_{x}=\frac{1}{2}\left(e^{2 \gamma_{Y}\left(x-x_{Y}\right)}-\zeta_{Y}\right) \quad ; \quad \zeta_{Y}=\operatorname{sign}\left(\tilde{c}_{Y} \gamma_{Y}\right)
$$


It is now straightforward to write down the complete solution

$$
\begin{aligned}
C_{x} & =e^{c_{C}^{Y} x+c_{C}^{Y^{\prime}}}\left(e^{\gamma_{Y} x_{Y}} K_{x}\right)^{-\frac{D-q_{Y}-3}{\Delta_{Y}}} \\
X_{x} & =e^{c_{X}^{Y} x+c_{X}^{Y^{\prime}}} e^{p_{X} g(x)}\left(e^{\gamma_{Y} x_{Y}} K_{x}\right)^{\frac{q_{Y}+1}{\Delta_{Y}}} \\
U_{x} & =e^{c_{U}^{Y} x+c_{U}^{Y^{\prime}}} e^{g(x)}\left(e^{\gamma_{Y} x_{Y}} K_{x}\right)^{\frac{q_{Y}+1}{\Delta_{Y}}} \\
Y_{x}=V_{x} & =e^{c_{Y}^{Y} x+c_{V}^{Y^{\prime}}}\left(e^{\gamma_{Y} x_{Y}} K_{x}\right)^{-\frac{D-q_{Y}-3}{\Delta_{Y}}} \\
W_{x} & =e^{c_{W}^{Y} x+c_{W}^{Y}}\left(e^{\gamma_{Y} x_{Y}} K_{x}\right)^{\frac{q_{Y}+1}{\Delta_{Y}}} \\
e^{\phi_{x}} & =e^{c_{\phi}^{Y} x+c_{\phi}^{Y^{\prime}}}\left(e^{\gamma_{Y} x_{Y}} K_{x}\right)^{\frac{(D-2) a_{Y} \epsilon_{Y}}{\Delta_{Y}}}
\end{aligned}
$$

where $c_{X}^{Y^{\prime}}=c_{U}^{Y^{\prime}}=\frac{-1}{p_{X}-1} \sum_{\alpha} c_{\alpha}^{Y^{\prime}}$. The constants $c_{\alpha}^{Y^{\prime}}=0$ and $e^{\gamma_{Y} x_{Y}}$ can be absorbed by a rescaling of the coordinates and we have done so in the main body of the paper and hereafter. The rescaling of $x$ and $y$ needed forces a compensating redefinition of all the constants $c_{\alpha}^{Y}$ and $c_{\alpha}^{X}$, including $\mu_{X}$ and $Q_{X}$. After doing so, an explicit calculation yields the following results

$$
\begin{aligned}
& S_{x}^{Y}=\frac{W_{x}^{p_{t}} U_{x}^{p_{X}}}{C_{x}^{p} X_{x} V_{x}^{p_{Y}+1}} e^{a_{Y} \phi_{x}}=e^{-2 \gamma_{Y} x} K_{x}^{2} \\
& S_{x}^{X}=\frac{W^{2 p_{t}} V^{2 p_{Y}}}{X_{x}^{2}} e^{a_{X} \phi_{x}}=e^{\kappa_{Y} x} K_{x}^{-\frac{2(D-2)}{\Delta_{Y}}}
\end{aligned}
$$

with $\kappa_{Y}=-\frac{2}{p_{X}-1}\left(p_{X} p_{t} c_{W}^{Y}+\left(p_{X} p_{Y}+1\right) c_{V}^{Y}+p c_{C}^{Y}\right)+a_{X} c_{\phi}^{Y}$. These functions are needed in order to solve for $E_{x}^{X}$

$$
E_{x}^{X}=\frac{l_{X}}{S_{x}^{X}}=l_{X} e^{\kappa_{Y} x} K_{x}^{\frac{2(D-2)}{\Delta_{Y}}}
$$

as well as to compute the normalization

$$
S_{x}^{Y} \partial_{x} E_{x}^{Y}=\operatorname{sign}\left(\tilde{c}_{Y} \gamma_{Y}\right) \frac{2(D-2) \gamma_{Y}^{2}}{\Delta_{Y} \tilde{c}_{Y}}=c_{Y}
$$

From here one readily gets the $R R$ charge of the $Y$ brane

$$
\mathcal{Q}_{Y}=\eta l_{Y} c_{Y} \Omega_{p_{Y}}=\eta \sqrt{\operatorname{sign}\left(c_{Y} \gamma_{Y}\right) \frac{2(D-2)}{\Delta_{Y}}} \gamma_{Y} \Omega_{p_{Y}}
$$

with $\eta=\eta_{T} \eta_{X} \eta_{Y}$. We see that the parameter $\gamma_{Y}$ is related to the charge and plays the rôle of $Q_{Y}$ in the other ansatz. Since $\zeta_{Y}=\operatorname{sign}\left(\tilde{c}_{Y} \gamma_{Y}\right)=\eta_{T} \eta_{Y} \operatorname{sign}\left(c_{Y} \gamma_{Y}\right)$, after choosing $\operatorname{sign}\left(c_{Y} \gamma_{Y}\right)=+1$ to ensure reality of the charge (B.21), we are allowed to replace $\zeta_{Y}=\eta_{T} \eta_{Y}$ in all the expressions. Now, for the gauge potential we obtain

$$
E_{x}^{Y}=-\frac{\eta_{T} \eta_{Y}}{l_{Y}} \sqrt{\frac{2(D-2)}{\Delta_{Y}}} \frac{e^{2 \gamma_{Y}\left(x-x_{Y}\right)}}{K_{x}}
$$


and we observe that the reality is independent of the signature choice. For the $y$ dependence we may find a specular solution after replacing $x \rightarrow y, U \leftrightarrow V$ and $Y \leftrightarrow X$. Alternatively, we may mix this ansatz with an ansatz of the form given for a black brane in the previous section. Then multiplying functions of $x$ with functions of $y$ the full solution is obtained.

\section{Bestiary of solutions}

In this appendix we shall give the explicit solutions for the intersection of black-branes $p_{X}, p_{Y} \neq 1$. The harmonic functions are the ones given in (3.6) and (3.7). The parameters $\xi^{A}, A=X, Y$. stand for

$$
\xi^{A}=\left(1+\frac{2 \mu_{A}}{Q_{A}}\right)^{-1 / 2} .
$$

First of all, the list of solutions that depend on three parameters contains the cases $(p-3|D p, N S 5| 1)$, $4 \leq p \leq 7$. Their actual expressions are a bit cumbersome so we are not going to include them, at least explicitly, in the bestiary.

\section{C.1 Solutions depending on two parameters}

Let us list the only five cases of two parameter (say, $\mu_{X}, \mu_{Y}$ ) family of solutions. As discussed in the body of the paper, for all these configurations we have found that one of the intervening branes is minimally non-extremal. We use $d \Omega_{k}^{2}$ for the round metric of the unit $k$-sphere.

\section{$(1|D 2, D 8| 0)$}

We find two solutions, depending on the intervening brane that is minimally non-extremal. When it is the D2-brane,

$$
\begin{aligned}
d s^{2} & =H_{x}^{9 / 8} H_{y}^{3 / 8} f_{x}^{-9 / 8}\left[H_{x}^{-1} H_{y}^{-1}\left(-f_{x} f_{y} d t^{2}+d z^{2}\right)+H_{y}^{-1} f_{x}^{-3} d x^{2}+H_{x}^{-1} f_{x}\left(f_{y}^{-1} d y^{2}+y^{2} d \Omega_{6}^{2}\right)\right] \\
e^{\phi} & =f_{x}^{5 / 4} H_{x}^{-5 / 4} H_{y}^{1 / 4} ; \quad E^{X}=\xi^{X} H_{x} f_{x}^{-3} H_{y}^{-1} f_{y} ; \quad E^{Y}=\xi^{Y} H_{x}^{-1} f_{x} y^{6} H_{y}
\end{aligned}
$$

whereas for the $D 6$-brane, it reads

$$
\begin{aligned}
d s^{2} & =H_{x}^{9 / 8} H_{y}^{3 / 8} f_{y}^{-3 / 8}\left[H_{x}^{-1} H_{y}^{-1}\left(-f_{x} f_{y} d t^{2}+d z^{2}\right)+H_{y}^{-1} f_{y}\left(f_{x}^{-1} d x^{2}\right)+H_{x}^{-1} f_{y}^{2 / 5}\left(f_{y}^{-1} d y^{2}+y^{2} d \Omega_{6}^{2}\right)\right] \\
e^{\phi} & =f_{y}^{-1 / 4} H_{x}^{-5 / 4} H_{y}^{1 / 4} ; E^{X}=\xi^{X} H_{x} f_{y} H_{y}^{-1} ; \quad E^{Y}=\xi^{Y} H_{x}^{-1} f_{x} y^{6} H_{y} f_{y}^{-3 / 5}
\end{aligned}
$$

$(1|D 4, D 6| 0)$

Again, we have two solutions according to whether it is the $D 4$-brane or the $D 6$-brane the minimally non-extremal one. When it is the D4-brane, we get

$$
\begin{gathered}
d s^{2}=H_{x}^{7 / 8} H_{y}^{5 / 8} f_{x}^{-7 / 8}\left[H_{x}^{-1} H_{y}^{-1}\left(-f_{x} f_{y} d t^{2}+d z^{2}\right)+H_{y}^{-1} f_{x}^{2}\left(f_{x}^{-1} d x^{2}+x^{2} d \Omega_{2}^{2}\right)\right. \\
\left.\quad+H_{x}^{-1} f_{x}\left(f_{y}^{-1} d y^{2}+y^{2} d \Omega_{4}^{2}\right)\right] \\
e^{\phi}=f_{x}^{3 / 4} H_{x}^{-3 / 4} H_{y}^{-1 / 4} ; \quad E^{X}=\xi^{X} x^{2} H_{x} f_{x} H_{y}^{-1} f_{y} ; \quad E^{Y}=\xi^{Y} H_{x}^{-1} f_{x} y^{4} H_{y}
\end{gathered}
$$


whereas for the $D 6$-brane, we obtain

$$
\begin{gathered}
d s^{2}=H_{x}^{7 / 8} H_{y}^{5 / 8} f_{y}^{-5 / 8}\left[H_{x}^{-1} H_{y}^{-1}\left(-f_{x} f_{y} d t^{2}+d z^{2}\right)+H_{y}^{-1} f_{y}\left(f_{x}^{-1} d x^{2}+x^{2} d \Omega_{2}^{2}\right)\right. \\
\left.+H_{x}^{-1} f_{y}^{2 / 3}\left(f_{y}^{-1} d y^{2}+y^{2} d \Omega_{4}^{2}\right)\right] \\
e^{\phi}=f_{y}^{1 / 4} H_{x}^{-3 / 4} H_{y}^{-1 / 4} ; E^{X}=\xi^{X} x^{2} H_{x} H_{y}^{-1} f_{y} ; \quad E^{Y}=\xi^{Y} H_{x}^{-1} f_{x} y^{4} H_{y} f_{y}^{-1 / 3}
\end{gathered}
$$

$(1|D 5, D 5| 0)$

We assume that the first $D 5$-brane is the minimally non-extremal one:

$$
\begin{aligned}
d s^{2}= & H_{x}^{3 / 4} H_{y}^{3 / 4} f_{x}^{-3 / 4}\left[H_{x}^{-1} H_{y}^{-1}\left(-f_{x} f_{y} d t^{2}+d z^{2}\right)+H_{y}^{-1} f_{x}\left(f_{x}^{-1} d x^{2}+x^{2} d \Omega_{3}^{2}\right)\right. \\
& \left.+\quad H_{x}^{-1} f_{x}\left(f_{y}^{-1} d y^{2}+y^{2} d \Omega_{3}^{2}\right)\right] \\
e^{\phi}= & f_{x}^{1 / 2} H_{x}^{-1 / 2} H_{y}^{-1 / 2} ; E^{X}=\xi^{X} x^{3} H_{x} H_{y}^{-1} f_{y} ; \quad E^{Y}=\xi^{Y} H_{x}^{-1} f_{x} y^{3} H_{y}
\end{aligned}
$$

The solution with $(x \leftrightarrow y)$ and $(X \leftrightarrow Y)$ just interchanges the rôle of the intervening $D 5$-branes.

$(1|N S 5, N S 5| 0)$

It is exactly as in the $(1|D 5, D 5| 0)$ solution except for the dilaton, $e^{\phi} \rightarrow e^{-\phi}$. Up to this point, notice that the solutions are fully localized.

$(1|M 5, M 5| 1)$

The only solution appearing in eleven dimensional supergravity. Taking the first M5-brane as the minimally non-extremal one, the solution reads

$$
\begin{gathered}
d s^{2}=H_{x}^{2 / 3} H_{y}^{2 / 3} f_{x}^{-2 / 3}\left[H_{x}^{-1} H_{y}^{-1}\left(-f_{x} f_{y} d t^{2}+d z^{2}\right)+H_{y}^{-1} f_{x}\left(f_{x}^{-1} d x^{2}+x^{2} d \Omega_{3}^{2}\right)\right. \\
\left.+H_{x}^{-1} f_{x}\left(f_{y}^{-1} d y^{2}+y^{2} d \Omega_{3}^{2}\right)+d w^{2}\right] \\
E^{X}=\xi^{X} x^{3} H_{x} H_{y}^{-1} f_{y} ; \quad E^{Y}=\xi^{Y} y^{3} H_{x}^{-1} f_{x} H_{y}
\end{gathered}
$$

Again, the solution with $(x \leftrightarrow y)$ and $(X \leftrightarrow Y)$ just interchanges the rôle of the $M 5$-branes.

\section{C.2 Solutions depending on one parameter}

Finally we list the solutions that depend on one parameter. They necessarily have either $\mu_{X}=0$ or $\mu_{Y}=0$, that is, one of the intervening branes is forced to be extremal.

$(0|D 1, D 7| 1)$

When the $D 1$-brane is extremal, $\mu_{X}=0$, and the $D 7$-brane can be minimally non-extremal

$$
\begin{aligned}
d s^{2} & =H_{x} H_{y}^{1 / 4}\left[-H_{x}^{-1} H_{y}^{-1} f_{x} d t^{2}+H_{y}^{-1} f_{x}^{-1} d x^{2}+H_{x}^{-1}\left(d y^{2}+y^{2} d \Omega_{6}^{2}\right)+d w^{2}\right] \\
e^{\phi} & =H_{x}^{-1} H_{y}^{1 / 2} ; \quad E^{X}=H_{x} H_{y}^{-1} ; \quad E^{Y}=\xi^{Y} H_{x}^{-1} f_{x} y^{6} H_{y}
\end{aligned}
$$


or a non-minimal non-extremal extension

$$
\begin{aligned}
d s^{2} & =H_{x} H_{y}^{1 / 4}\left[-H_{x}^{-1} H_{y}^{-1} f_{x} d t^{2}+H_{y}^{-1} f_{x}^{114 / 7} d x^{2}+f_{x}^{22 / 7} H_{x}^{-1}\left(d y^{2}+y^{2} d \Omega_{6}^{2}\right)+f_{x}^{-33 / 7} d w^{2}\right] \\
e^{\phi} & =f_{x}^{-11} H_{x}^{-1} H_{y}^{1 / 2} ; \quad E^{X}=H_{x} f_{x}^{99 / 7} H_{y}^{-1} ; E^{Y}=\xi^{Y} H_{x}^{-1} f_{x} y^{6} H_{y}
\end{aligned}
$$

When it is the $D 7$-brane the extremal one, $\mu_{Y}=0$, the $D 1$-brane might be minimally non-extremal

$$
\begin{aligned}
d s^{2} & =H_{x} H_{y}^{1 / 4}\left[-H_{x}^{-1} H_{y}^{-1} f_{y} d t^{2}+H_{y}^{-1} d x^{2}+H_{x}^{-1}\left(f_{y} d y^{2}+y^{2} d \Omega_{6}^{2}\right)+d w^{2}\right] \\
e^{\phi} & =H_{x}^{-1} H_{y}^{1 / 2} ; \quad E^{X}=\xi^{X} H_{x} H_{y}^{-1} f_{y} ; \quad E^{Y}=H_{x}^{-1} y^{6} H_{y}
\end{aligned}
$$

or a non-minimal non-extremal extension

$$
\begin{aligned}
d s^{2} & =H_{x} H_{y}^{1 / 4}\left[-H_{x}^{-1} H_{y}^{-1} f_{y} d t^{2}+f_{y}^{-2 / 19} H_{y}^{-1} d x^{2}+f_{y}^{8 / 95} H_{x}^{-1}\left(f_{y} d y^{2}+y^{2} d \Omega_{6}^{2}\right)+f_{y}^{-6 / 19} d w^{2}\right] \\
e^{\phi} & =H_{x}^{-1} H_{y}^{1 / 2} f_{y}^{-2 / 19} ; \quad E^{X}=\xi^{X} H_{x} H_{y}^{-1} f_{y} ; \quad E^{Y}=H_{x}^{-1} y^{6} H_{y} f_{y}^{18 / 95}
\end{aligned}
$$

$(0|D 3, D 5| 1)$

When the $D 3$-brane is extremal, $\mu_{X}=0$, the $D 5$-brane can be minimally non-extremal

$$
\begin{aligned}
d s^{2} & =H_{x}^{3 / 4} H_{y}^{1 / 2}\left[-H_{x}^{-1} H_{y}^{-1} f_{x} d t^{2}+H_{y}^{-1}\left(f_{x}^{-1} d x^{2}+x^{2} d \Omega_{2}^{2}\right)+H_{x}^{-1}\left(d y^{2}+y^{2} d \Omega_{4}^{2}\right)+d w^{2}\right] \\
e^{\phi} & =H_{x}^{-1 / 2} ; \quad E^{X}=x^{2} H_{x} H_{y}^{-1} ; \quad E^{Y}=\xi^{Y} H_{x}^{-1} f_{x} y^{4} H_{y}
\end{aligned}
$$

or a non-minimal non-extremal extension

$$
\begin{aligned}
d s^{2}= & H_{x}^{3 / 4} H_{y}^{1 / 2}\left[-H_{x}^{-1} H_{y}^{-1} f_{x} d t^{2}+H_{y}^{-1} f_{x}^{2 / 17}\left(f_{x}^{-1} d x^{2}+x^{2} d \Omega_{2}^{2}\right)+H_{x}^{-1} f_{x}^{-1 / 17}\left(d y^{2}+y^{2} d \Omega_{4}^{2}\right)\right. \\
& \left.+f_{x}^{3 / 17} d w^{2}\right] \\
e^{\phi}= & H_{x}^{-1 / 2} f_{x}^{5 / 17} ; \quad E^{X}=x^{2} H_{x} f_{x}^{3 / 17} H_{y}^{-1} ; \quad E^{Y}=\xi^{Y} H_{x}^{-1} f_{x} y^{4} H_{y}
\end{aligned}
$$

Instead, when it is the $D 5$-brane the extremal one, $\mu_{Y}=0$, the only solution has a minimally non-extremal D3-brane

$$
\begin{aligned}
d s^{2} & =H_{x}^{3 / 4} H_{y}^{1 / 2}\left[-H_{x}^{-1} H_{y}^{-1} f_{y} d t^{2}+H_{y}^{-1}\left(d x^{2}+x^{2} d \Omega_{2}^{2}\right)+H_{x}^{-1}\left(f_{y}^{-1} d y^{2}+y^{2} d \Omega_{4}^{2}\right)+d w^{2}\right] \\
e^{\phi} & =H_{x}^{-1 / 2} ; \quad E^{X}=\xi^{X} x^{2} H_{x} H_{y}^{-1} f_{y} ; \quad E^{Y}=H_{x}^{-1} y^{4} H_{y}
\end{aligned}
$$

$(0|D 3, N S 5| 1)$

It is exactly as in the previous case except for the dilaton, $e^{\phi} \rightarrow e^{-\phi}$.

$(0|D 4, D 4| 1)$

Let us consider that the first $D 4$-brane is the extremal one, $\mu_{X}=0$. Then, we have two solutions according to whether the other one is minimally non-extremal

$$
\begin{aligned}
d s^{2} & =H_{x}^{5 / 8} H_{y}^{5 / 8}\left[-H_{x}^{-1} H_{y}^{-1} f_{x} d t^{2}+H_{y}^{-1}\left(f_{x}^{-1} d x^{2}+x^{2} d \Omega_{3}^{2}\right)+H_{x}^{-1}\left(d y^{2}+y^{2} d \Omega_{3}^{2}\right)+d w^{2}\right] \\
e^{\phi} & =H_{x}^{-1 / 4} H_{y}^{-1 / 4} ; \quad E^{X}=x^{3} H_{x} H_{y}^{-1} ; E^{Y}=\xi^{Y} H_{x}^{-1} f_{x} y^{3} H_{y}
\end{aligned}
$$


or a non-minimal non-extremal extension

$$
\begin{gathered}
d s^{2}=H_{x}^{5 / 8} H_{y}^{5 / 8}\left[-H_{x}^{-1} H_{y}^{-1} f_{x} d t^{2}+H_{y}^{-1} f_{x}^{1 / 85}\left(f_{x}^{-1} d x^{2}+x^{2} d \Omega_{3}^{2}\right)+H_{x}^{-1} f_{x}^{1 / 85}\left(d y^{2}+y^{2} d \Omega_{3}^{2}\right)\right. \\
\left.\quad+f_{x}^{-6 / 85} d w^{2}\right] \\
e^{\phi}=f_{x}^{-8 / 85} H_{x}^{-1 / 4} H_{y}^{-1 / 4} ; E^{X}=x^{3} H_{x} H_{y}^{-1} ; E^{Y}=\xi^{Y} H_{x}^{-1} f_{x} y^{3} H_{y}
\end{gathered}
$$

\section{References}

[1] A. Sen, Non-BPS states and branes in string theory, [arXiv:hep-th/9904207].

[2] M. Gutperle and A. Strominger, Spacelike branes, JHEP 0204 (2002) 018 [arXiv:hep-th/0202210].

[3] C. M. Chen, D. V. Gal'tsov and M. Gutperle, S-brane solutions in supergravity theories, Phys. Rev. D 66 (2002) 024043 [arXiv:hep-th/0204071].

[4] M. Kruczenski, R. C. Myers and A. W. Peet, Supergravity S-branes, JHEP 0205 (2002) 039 [arXiv:hepth/0204144].

[5] S. Roy, On Supergravity Solutions of space-like Dp branes, JHEP 0208 (2002) 025 [arXiv:hepth/0205198]. S. Bhattacharya and S. Roy, Time Dependent Supergravity Solutions in Arbitrary Dimensions, JHEP 0312 (2003) 015 [arXiv:hep-th/0309202].

[6] A. Buchel, P. Langfelder and J. Walcher, On time dependent backgrounds in Supergravity and String Theory, Phys. Rev. D 67 (2003) 024011 [arXiv:hep-th/0207214]. Does The Tachyon Matter, Annals Phys. 302 (2002) 78 [arXiv:hep-th/0207235].

[7] A. Buchel and J. Walcher, Comments on Supergravity Description of S-branes, JHEP 0305 (2003) 069 [arXiv:hep-th/0305055]. The Tachyon Does Matter, Fortsch. Phys. 51 (2003) 885 [arXiv:hepth/0212150].

[8] F. Leblond and A. Peet, D-brane gravity fields and rolling tachyons JHEP 0304 (2003) 048 [arXiv:hepth/0303035].

[9] G. Jones, A. Maloney and A. Strominger, Non-Singular Solutions for S-branes, [arXiv:hepth/0403050]. J. E. Wang, Twisting S-branes, [arXiv:hep-th/0403094]. G. Tasinato, I. Zavala, C. P. Burguess and F. Quevedo, Regular S-Brane Backgrounds, [arXiv:hep-th/0403156].

[10] J. Demaret, J. L. Hanquin, M. Henneaux and P. Spindel, Cosmological Models In Eleven-Dimensional Supergravity, Nucl. Phys. B 252 (1985) 538. K. Behrndt and S. Forste, String Kaluza-Klein cosmology, Nucl. Phys. B 430 (1994) 441 [arXiv:hep-th/9403179]. A. Lukas, B. A. Ovrut and D. Waldram, Cosmological solutions of type II string theory, Phys. Lett. B 393 (1997) 65 [arXiv:hep-th/9608195]. String and M-theory cosmological solutions with Ramond forms, Nucl. Phys. B 495 (1997) 365 [arXiv:hepth/9610238]. H. Lu, S. Mukherji, C. N. Pope and K. W. Xu, Cosmological solutions in string theories, Phys. Rev. D 55 (1997) 7926 [arXiv:hep-th/9610107]. H. Lu, S. Mukherji and C. N. Pope, From p-branes to cosmology, Int. J. Mod. Phys. A 14 (1999) 4121 [arXiv:hep-th/9612224]. 
[11] C. Grojean, F. Quevedo, G. Tasinato and I. Zavala. Branes on charged dilatonic backgrounds: Selftuning, Lorentz violations and cosmology, JHEP 0108(2001)005 [arXiv:hep-th/0106120]. C. Burgess, F. Quevedo, S. J. Rey, G. Tasinato and I. Zavala. Cosmological spacetimes from negative tension brane backgrounds JHEP 0210 (2002) 028 [arXiv:hep-th/0207104].

[12] L. Cornalba and M. S. Costa, A new cosmological scenario in string theory, Phys. Rev. D 66 (2002) 066001 [arXiv:hep-th/0203031]. Time-dependent orbifolds and string cosmology, Fortsch. Phys. 52 (2004) 145 [arXiv:hep-th/0310099], and references therein.

[13] G. W. Gibbons, Aspects Of Supergravity Theories, in "Supersymmetry, Supergravity and Related Topics", edited by F. de Aguila, J.A. de Azcárraga and L. Ibañez, World Scientific, Singapore (1985) 346. J. M. Maldacena and C. Núñez, Supergravity description of field theories on curved manifolds and a no go theorem, Int. J. Mod. Phys. A 16 (2001) 822 [arXiv:hep-th/0007018].

[14] P. K. Townsend and M. N. R. Wohlfarth, Accelerating cosmologies from compactification, Phys. Rev. Lett. 91 (2003) 061302 [arXiv:hep-th/0303097].

[15] N. Ohta, Accelerating cosmologies from S-branes, Phys. Rev. Lett. 91, 061303 (2003), [arXiv:hepth/0303238]. A study of accelerating cosmologies from superstring / $M$ theories, Prog. Theor. Phys. 110 (2003) 269 [arXiv:hep-th/0304172].

[16] S. Roy, Accelerating cosmologies from M/string theory compactifications, Phys. Lett. B 567 (2003) 322 [arXiv:hep-th/0304084].

[17] N. Ohta, Intersection rules for S-branes, Phys. Lett. B 558 (2003) 213 [arXiv:hep-th/0301095].

[18] N. S. Deger and A. Kaya, Intersecting S-brane solutions of D = 11 supergravity, JHEP 0207 (2002) 038 [arXiv:hep-th/0206057]. V. D. Ivashchuk, Composite S-brane solutions related to Toda-type systems, Class. Quant. Grav. 20 (2003) 261 [arXiv:hep-th/0208101]. N. S. Deger, Non-standard intersections of S-branes in $D=11$ supergravity, JHEP 0304 (2003) 034 [arXiv:hep-th/0303232]. V. D. Ivashchuk, On composite S-brane solutions with orthogonal intersection rules, arXiv:hep-th/0309027.

[19] R. Argurio, F. Englert and L. Houart, Intersection rules for p-branes, Phys. Lett. B 398 (1997) 61 [arXiv:hep-th/9701042]. For a review and an exhaustive list of references see for example: J. P. Gauntlett, Intersecting Branes, [arXiv:hep-th/9705011]. R. Argurio, Brane physics in M-theory, [arXiv:hep-th/9807171]. K. Skenderis, Black holes and branes in string theory, Lect. Notes Phys. 541 (2000) 325 [arXiv:hep-th/9901050]. D. J. Smith, Intersecting Brane Solutions in String and M-Theory, Class. Quant. Grav. 20 (2003) R233 [arXiv:hep-th/0210157].

[20] M. Cvetic and A. A. Tseytlin, Non-extremal black holes from non-extreme intersecting M-branes, Nucl. Phys. B 478 (1996) 431 [arXiv:hep-th/9606033].

[21] I. Y. Aref'eva, M. G. Ivanov and I. V. Volovich, Non-extremal intersecting p-branes in various dimensions, Phys. Lett. B 406 (1997) 44 [arXiv:hep-th/9702079].

[22] N. Ohta, Intersection Rules for Non-Extreme p-Branes, Phys. Lett. B 403 (1997) 218 [arXiv:hepth/9702095]. 
[23] R. R. Khuri, A Comment on String Solitons Phys. Rev. D 48 (1993) 2947 [arXiv:hep-th/9305143]. J. P. Gauntlett, D. A. Kastor and J. Traschen. Overlapping Branes in M-Theory, Nucl. Phys. B 478 (1996) 544 [arXiv:hep-th/9604179]. A. A. Tseytlin, Composite BPS configurations of p-branes in 10 and 11 dimensions, Class. Quant. Grav. 14 (1997) 2085 [arXiv:hep-th/9702163].

[24] J. D. Edelstein, L. Tataru and R. Tatar, Rules for localized overlappings and intersections of p-branes, JHEP 9806 (1998) 003 [arXiv:hep-th/9801049].

[25] J. P. Gauntlett, R. C. Myers and P. K. Townsend Supersymmetry of Rotating Branes Phys. Rev. D 59 (1999) 025001 [arXiv:hep-th/9809065]. A. Fayyazuddin and D. J. Smith Localized intersections of M5-branes and four-dimensional superconformal field theories JHEP 9904 (1999) 030 [arXiv:hepth/9902210]. D. Youm Partially Localized Intersecting BPS Branes [arXiv:hep-th/9902208], Supergravity Solutions for BI Dyons Phys. Rev. D 60 (1999) 105006 [arXiv:hep-th/9905155]. A. Loewy, Semi Localized Brane Intersections in SUGRA, Phys. Lett. B 463 (1999) 41 [arXiv:hep-th/9903038].

[26] I. Y. Aref'eva, M. G. Ivanov, O. A. Rytchkov and I. V. Volovich, Non-extremal localized branes and vacuum solutions in M-theory, Class. Quant. Grav. 15 (1998) 2923 [arXiv:hep-th/9802163].

[27] C. M. Hull, Timelike T-duality, de Sitter space, large $N$ gauge theories and topological field theory, JHEP 9807 (1998) 021 [arXiv:hep-th/9806146].

[28] B. Zhou and C.-J. Zhu, The Complete Black Brane Solutions in D-dimensional Coupled Gravity System [arXiv:hep-th/9905146].

[29] V. D. Ivashchuk and V. N. Melnikov, Exact solutions in multidimensional gravity with antisymmetric forms, Class. Quant. Grav. 18 (2001) R87 [arXiv:hep-th/0110274], and references therein.

[30] S. de Haro, A. Sinkovics and K. Skenderis, On alpha'-corrections to D-brane solutions, Phys. Rev. D 68 (2003) 066001 [arXiv:hep-th/0302136].

[31] P. Brax, G. Mandal and Y. Oz, Supergravity Description of Non-BPS Branes Phys. Rev. D 63 (2001) 064008 [arXiv:hep-th/0005242].

[32] D. Gal'tsov, J. P. S. Lemos and G. Clément, Supergravity p-branes revisited: extra parameters, uniqueness, and topological censorship [arXiv:hep-th/0403112].

[33] J. X. Lu and S. Roy, Supergravity approach to tachyon condensation on the brane-antibrane system, [arXiv:hep-th/0403147].

[34] C. M. Chen, P. M. Ho, I. P. Neupane and J. E. Wang, A note on acceleration from product space compactification, JHEP 0307 (2003) 017, [arXiv:hep-th/0304177]. If, instead, all factors are hyperbolic, the number of e-foldings approaches one: C. M. Chen, P. M. Ho, I. P. Neupane, N. Ohta and J. E. Wang, Hyperbolic space cosmologies, JHEP 0310 (2003) 058 [arXiv:hep-th/0306291].

[35] R. Emparan and J. Garriga, A note on accelerating cosmologies from compactifications and S-branes, JHEP 0305 (2003) 028 [arXiv:hep-th/0304124].

[36] J. G. Russo, Exact solution of scalar-tensor cosmology with exponential potentials and transient acceleration, [arXiv:hep-th/0403010]. 
[37] I. P. Neupane, Accelerating cosmologies from exponential potentials, [arXiv:hep-th/0311071]. P. G. Vieira, Late-time cosmic dynamics from M-theory, [arXiv:hep-th/0311173].

[38] J. D. Edelstein and J. Mas, in preparation.

[39] F. Leblond, Mirage resolution of cosmological singularities, [arXiv:hep-th/0403221]. 